\title{
The Dirac Sea, $T$ and $C$ Symmetry Breaking, and the Spinor Vacuum of the Universe ${ }^{+}$
}

\author{
Vadim Monakhov (D)
}

check for

updates

Citation: Monakhov, V. The Dirac

Sea, $T$ and $C$ Symmetry Breaking, and the Spinor Vacuum of the Universe ${ }^{\dagger}$. Universe 2021, 7, 124. https:// doi.org/10.3390/universe7050124

Academic Editors: James A. Isenberg, Gerald B. Cleaver, Lijing Shao,

Gonzalo J. Olmo and

Giacomo Tommei

Received: 29 March 2021

Accepted: 27 April 2021

Published: 1 May 2021

Publisher's Note: MDPI stays neutral with regard to jurisdictional claims in published maps and institutional affiliations.

Copyright: (C) 2021 by the author. Licensee MDPI, Basel, Switzerland. This article is an open access article distributed under the terms and conditions of the Creative Commons Attribution (CC BY) license (https:/ / creativecommons.org/licenses/by/ $4.0 /)$.
Department of Computational Physics, Faculty of Physics, Saint Petersburg State University, Ulyanovskaya Street 1, 198504 Saint Petersburg, Russia; v.v.monahov@spbu.ru

† This paper is an extended version from the proceeding paper: Vadim Monakhov. T and C Symmetry Breaking in Algebraic Quantum Field Theory. In Proceedings of the 1st Electronic Conference on Universe, online, 22-28 February 2021.

\begin{abstract}
We have developed a quantum field theory of spinors based on the algebra of canonical anticommutation relations (CAR algebra) of Grassmann densities in the momentum space. We have proven the existence of two spinor vacua. Operators $C$ and $T$ transform the normal vacuum into an alternative one, which leads to the breaking of the $C$ and $T$ symmetries. The $C P T$ is the real structure operator; it preserves the normal vacuum. We have proven that, in the theory of the Dirac Sea, the formula for the charge conjugation operator must contain an additional generalized Dirac conjugation operator.
\end{abstract}

Keywords: CAR algebra; Clifford algebra; Krein spaces; discrete symmetries; Dirac Sea; time reversal; charge conjugation; CPT; vacuum state; alternative vacuum

\section{Introduction}

The quantum mechanical time inversion operator was proposed by Kramers [1] and Wigner [2]. The operator of charge conjugation was proposed by Kramers [3] in the framework of the theory of "holes" in the Dirac Sea of electrons with negative energy. It was an antiunitary operator that preserved the form of the Dirac equation. Later, the method of deriving symmetry transformation formulas based on preserving the Dirac equation was used by many authors to derive formulas not only for the operator of charge conjugation $C$ but also for the $P$ and $T$ operators of space and time inversion [4-6]. We also use this approach. Paper [3] served as the basis for the development of the theory of discrete symmetries. However, such a definition of the charge conjugation led to the fact that the charge conjugation operator was later regarded as antiunitary by Schwinger [7], Pauli [8], Bell [9], Grawert, Lüders and Rollnik [5], Bjorken and Drell [6,10], Berestetskii [11], Weinberg [12], and others. Whereas, in some publications considered classical, it was declared that the operator of charge conjugation of spinors is unitary, in the formulas it acted as antiunitary $[5,6,10,12-14]$. Below, we prove that, in the framework of the theory of "holes" in the Dirac Sea, the procedure of replacing "holes" in the Dirac Sea with antiparticles should be considered as an antiunitary operator. Therefore, a correctly defined operator of the charge conjugation in the theory of "holes" is unitary.

The foundations of the relativistic covariant formulation of the quantum mechanics (relativistic quantum field theory-RQFT) for fermions were laid down by Julian Schwinger. He proposed a Lorentz covariant interpretation of the spatial inversion (operator $P$ ), the time inversion (operator $T$ ), and the charge conjugation (operator $C$ ) $[7,15]$. He also showed that in RQFT the time inversion operator must be antiunitary $[7,15]$, whereas both Schwinger [7,15] and Bell [9] considered the time inversion operator as the operator that also changes the charge to the opposite one. Moreover, Schwinger proposed another time inversion operator that was unitary [15]. It contained transposition and changed the ket 
vectors to the bra vectors. All this further introduced additional confusion on the issue of operators of the charge conjugation and the time inversion.

The idea of the CPT transformation was first implicitly formulated by Schwinger [15] and Lüders [16]. Schwinger showed that, for $P$-invariant theories, the correct statistics for particles with half-integer spin follows from the requirement of simultaneous time inversion and charge conjugation [15]. Lüders showed that, in an arbitrary $P$-invariant theory in which the anticommutation rules are satisfied for particles with half-integer spin, the CT symmetry holds [16]. Additionally, they formulated a modern algebraic approach to the description of antiparticles. In this approach, field operators of spinors and antispinors participate equally, and there is no use of the concept of the Dirac Sea.

The first explicit formulation of the existence of the CPT symmetry belongs to Pauli [8]. However, at that time, this symmetry had not yet received its modern name. Pauli referred to the CPT transformation as "strong reflection". Similar to Kramers, he considered the negative frequency parts of the electron wave function within the framework of the concept of "holes" in the Dirac Sea consisting of electrons with negative mass as positive frequency parts of the positron wave function. This interpretation and the corresponding formulas for charge conjugation have become standard $[6,10,17]$.

The idea of the CPT symmetry (also called TCP, CTP, $P C T$, etc.) was formulated as a theorem by Lüders [4] following the suggestion of Pauli. This theorem states that "Local quantum field theory, invariant with respect to the Lorentz transformations and including the usual causal commutativity or anticommutativity of the field operators, is always invariant under the product of the T, $C$ and $P$ transformations" [5]. Moreover, all processes in the CPT coupled world occur with the same probabilities, and the Lagrangian of the system is a Hermitian operator that is invariant with respect to the CPT transformation. In particular, following the extension of Landau's idea of combined symmetry [18], if there exists a field operator $\Psi$ corresponding to a fermion particle, then it corresponds to the $C P T$ transformed field operator $C P T \Psi$ of an antiparticle that actually exists in nature and has equal mass and opposite charges of all existing types [5]. The fact that the CPT operator commutes with the Hamiltonian implies the equality of masses of particles and antiparticles [12]. This transformation connects the S-matrix of an arbitrary process with the S-matrix of the inverse process (time inversion), in which the sign of the spin projections is opposite and the particles are replaced with antiparticles [12,19].

The proofs of the CPT theorem have been considered by Pauli [8], Lüders [4], Gravert, Lüders and Rolnik [5], Jost [20,21], Streater and Wightman [22], Epstein [19], Bjorken and Drell [6,10], Wess [23], Weinberg [12], Greenberg [24], Peskin and Schroeder [14], and others. Within the framework of the axiomatic field theory, Jost succeeded in carrying out the mathematically rigorous proof of the CPT theorem based on the properties of the Wightman functions in the complex Lorentz group [20,21,24]. It was considered a single transformation and not a product of separate transformations $C, P$, and $T$. This proof significantly reduced the number of uncertainties in the issues of the $C, P$, and $T$ transformations.

Initially, the $C P T$ theorem was a purely academic subject since the $P, T$, and $C$ symmetries were considered to be satisfied independently. However, after the discovery of the violation of the $P$ symmetry, interest in the CPT symmetry noticeably increased [5]. The $C P$ symmetry was proposed in 1957 by Landau as a true symmetry between matter and antimatter [18]. The discovery in 1964 of the $C P$ symmetry breaking in the decay processes of neutral K-mesons [25] led to the fact that, at present, the CPT symmetry, along with local Lorentz covariance, are considered to be the fundamental principles of the quantum field theory [24]. The real Lorentz group can be taken as the group $O(1,3)$ with four independent connected components. Only one of them (proper orthochronous) contains the identity and is, therefore, a group. For the complex Lorentz group, there are only two independent connected components. $P$ and $T$ are the transformations of the Lorentz group that do not lie in a connected neighborhood of the identity operator. The CPT transformation is a transformation of the complex Lorentz group that lies in a connected neighborhood of the 
identity [24]. The CPT conservation and Lorentz symmetry together present the group $O(1,3, \mathbb{C})$.

A number of unresolved issues still remain in the mentioned area. The study of the possible violation of the $C P$ and the $T$ symmetries in the lepton sector using the experiments on neutrino oscillations is one of the most important directions in the modern physics of quantum fields [26,27]. The possibility of the CPT symmetry breaking and the connection of such breaking with the $C P$ and the $T$ symmetry breaking and possible local Lorentz covariance breaking are also actively discussed and investigated [28,29].

In the area of discrete spinor symmetries, erroneous interpretations are somehow related to the concept of the Dirac sea, including the description of the spinor vacuum. In particular, all authors who studied the $P, T$, and $C$ transformations assumed that the vacuum is invariant with respect to these transformations. As we show below, this is not always the case. In the axiomatic field theory $[20,24]$, it was also assumed that the vacuum is invariant with respect to the $C P T$ transformation. However, as we prove below, the vacuum is actually invariant with respect to the $C P T$ transformation, and on this point of view, Jost's proof is correct. It does not consider separately the $T$ and $C$ transformations. However, considering separately the time inversion $T$ and the charge conjugation $C$ requires taking into account the replacement of the vacuum by an alternative one.

The meaning of the unitary time inversion operator proposed by Schwinger [15] has not yet been clarified. This operator is considered in more detail by Matthews [13]. There is still no justification of why it is necessary to use a different time inversion operator in the $C P T$ theorem.

In view of the above, the study of the algebraic foundations of the $C, P, T$, and $C P T$ symmetries and the properties of the spinor vacuum with respect to these transformations seems to be an important problem.

In this paper, we consider the $C, P, T$, and $C P T$ transformations for spinors using a purely algebraic approach within the framework of the theory of superalgebraic representation of spinors. It combines the theory of the CAR algebras and the theory of Krein spaces, a rapidly developing part of the theory of Clifford algebras. The correct formula for the charge conjugation operator in the theory of "holes" in the Dirac Sea is derived. We explain below the difference between the time reflection and time inversion operators and why this Schwinger operator cannot be the time inversion operator. We also show that the existence of the exact $C$ and $T$ symmetries is impossible for spinors. This violation occurs due to the fact that the $C$ and $T$ transformations replace the spinor vacuum of the Universe with an alternative vacuum.

\section{Superalgebraic Spinors}

Initially, in the quantum field theory, spinors were considered mainly in the framework of the Dirac matrix theory of spinors. The modern theory of spinors is usually formulated within the framework of the Clifford algebra formalism [30-39]. Such spinors are called algebraic. They are elements of the minimal left ideal formed by multiplying all elements of the Clifford algebra by a primitive Hermitian idempotent. Nevertheless, algebraic spinors are still rarely used in the quantum field theory. Additionally, in these theories, the creation and annihilation operators of spinors are optional external algebraic constructions that are in no way connected with the algebraic spinors themselves.

Additional problems in the theory of the algebraic spinors are related to the theory of spinor bundles. In the general case, automorphisms of the Clifford algebra do not preserve its spinor spaces [31]. In the odd-dimensional cases, the group of automorphisms of the Clifford algebra is not the group of automorphisms of the spinor bundle. In the even-dimensional case, all automorphisms of the Clifford algebra are inner and preserve the space of spinors. Only the even-dimensional spaces are physically meaningful, where spinors can exist in the curved spacetime. Therefore, we consider only the even-dimensional spaces with dimension $n=2 m$, where $m$ is integer. 
M. Pavšic, in the paper [38], laid the foundations for the theory of superalgebraic spinors, which combined the theory of the algebraic spinors and the algebraic quantum field theory. He tried to build a vacuum state vector of the operators of creation and annihilation of spinors. In this approach, the basis Clifford spacetime vectors (algebraic analogs of Dirac matrices) consist of the creation and the annihilation operators of spinors. However, he did not take into account that two different Clifford algebras should exist in the theory of the second quantization of fermions. In the first of them, as considered in [38], Clifford basis vectors with a positive signature are the sums of the creation and annihilation operators of a fermion with a given momentum, while vectors with a negative signature are the differences between them

$$
\begin{aligned}
& e_{i+}(p)=\bar{b}_{i}(p)+b_{i}(p), \\
& e_{i-}(p)=\bar{b}_{i}(p)-b_{i}(p) .
\end{aligned}
$$

We called this algebra the big Clifford algebra and the algebra of the algebraic analogs of the Dirac gamma matrices the small Clifford algebra [40].

The theory of superalgebraic spinors [40-44] develops the approach proposed in [38]. In it, spinors, basis vectors of the small Clifford algebra, and the vacuum state vector consist of Grassmann variables and their derivatives. In this case, the second quantization expansion for the spinor field operator is obtained from purely algebraic relations.

The theory of algebraic spinors is not based on the canonical anticommutation relations (CAR), although the modern formulation of the algebraic quantum field theory is based on them. In the theory of superalgebraic spinors, we used the CAR as the basis for deriving the formulas for the generalized Dirac conjugation [42]. However, the CAR were considered not as the basis of the theory but only as one of its elements. In this paper, we consider the CAR algebra as the basis for deriving all transformations in the theory of spinors.

The superalgebraic representation of spinors [40-44] can be described as a representation of the CAR algebra. This approach allows us to solve a number of problems in the algebraic quantum field theory. Instead of considering abstract CAR algebras [45], it turns out to be possible, without loss of generality, to construct concrete algebraic representations of these algebras.

In the superalgebraic representation of the 4-spinors, the basis spinors are Grassmann densities $\theta^{a}(p)$ in the momentum space and their derivatives $\frac{\partial}{\partial \theta^{a}(p)}, a=1,2,3,4$. They are used to construct anticommuting gamma operators $\hat{\gamma}^{\mu}=\left[\gamma^{\mu}, \bullet\right], \mu=0,1,2,3,5$, which are algebraic analogs of the Dirac matrices $\gamma_{D}^{\mu}[40,41]$, where

$$
\begin{gathered}
\hat{\gamma}^{0}=\int \mathrm{d}^{3} p\left[\frac{\partial}{\partial \theta^{1}(p)} \theta^{1}(p)+\frac{\partial}{\partial \theta^{2}(p)} \theta^{2}(p)+\frac{\partial}{\partial \theta^{3}(p)} \theta^{3}(p)+\frac{\partial}{\partial \theta^{4}(p)} \theta^{4}(p), \bullet\right], \\
\hat{\gamma}^{1}=\int \mathrm{d}^{3} p\left[\frac{\partial}{\partial \theta^{1}(p)} \frac{\partial}{\partial \theta^{4}(p)}-\theta^{4}(p) \theta^{1}(p)+\frac{\partial}{\partial \theta^{2}(p)} \frac{\partial}{\partial \theta^{3}(p)}-\theta^{3}(p) \theta^{2}(p), \bullet\right], \\
\hat{\gamma}^{2}=i \int \mathrm{d}^{3} p\left[-\frac{\partial}{\partial \theta^{1}(p)} \frac{\partial}{\partial \theta^{4}(p)}-\theta^{4}(p) \theta^{1}(p)+\frac{\partial}{\partial \theta^{2}(p)} \frac{\partial}{\partial \theta^{3}(p)}+\theta^{3}(p) \theta^{2}(p), \bullet\right], \\
\hat{\gamma}^{3}=\int \mathrm{d}^{3} p\left[\frac{\partial}{\partial \theta^{1}(p)} \frac{\partial}{\partial \theta^{3}(p)}-\theta^{3}(p) \theta^{1}(p)-\frac{\partial}{\partial \theta^{2}(p)} \frac{\partial}{\partial \theta^{4}(p)}+\theta^{4}(p) \theta^{2}(p), \bullet\right], \\
\hat{\gamma}^{5}=\int \mathrm{d}^{3} p\left[\frac{\partial}{\partial \theta^{1}(p)} \frac{\partial}{\partial \theta^{3}(p)}+\theta^{3}(p) \theta^{1}(p)+\frac{\partial}{\partial \theta^{2}(p)} \frac{\partial}{\partial \theta^{4}(p)}+\theta^{4}(p) \theta^{2}(p), \bullet\right] .
\end{gathered}
$$

Operator

$$
\hat{A}=[A, \bullet]
$$

denotes commutator

$$
\hat{A} \Psi=[A, \bullet] \Psi=[A, \Psi]=A \Psi-\Psi A .
$$

An arbitrary spinor $\Psi$ is a linear combination of the basis spinors

$$
\Psi=\int d^{3} p\left(\psi^{\alpha}(p) \frac{\partial}{\partial \theta^{\alpha}(p)}+\psi^{\tau}(p) \theta^{\tau}(p)\right),
$$

where $\psi^{a}(p)$ are complex coefficients, $\alpha=1,2 ; \tau=3,4$. 
The normalization condition

$$
|\Psi|^{2}=\int d^{3} p\left(\left|\psi^{\alpha}(p)\right|^{2}+\left|\psi^{\tau}(p)\right|^{2}\right)=1 .
$$

Canonical anticommutation relations (CAR)

$$
\begin{gathered}
\left\{\frac{\partial}{\partial \theta^{\alpha}(p)}, \theta^{\beta}\left(p^{\prime}\right)\right\}=\delta_{\alpha}^{\beta} \delta\left(p-p^{\prime}\right), \\
\left\{\frac{\partial}{\partial \theta^{\alpha}(p)}, \frac{\partial}{\partial \theta^{\beta}\left(p^{\prime}\right)}\right\}=\left\{\theta^{\alpha}(p), \theta^{\beta}\left(p^{\prime}\right)\right\}=0,
\end{gathered}
$$

define the CAR algebra of Grassmann densities.

The rule (9) of the Hermitian conjugation follows immediately from (4)

$$
(\hat{A} \Psi)^{+}=(A \Psi-\Psi A)^{+}=-A^{+} \Psi^{+}+\Psi^{+} A^{+}=-\left(A^{+}\right) \Psi^{+} .
$$

It was shown in [41] that if $\hat{A}$ is gamma operator, then

$$
\left(A^{+}\right)^{\wedge}=\hat{A}^{+} \text {. }
$$

the generalized Dirac conjugation is given by the formula $[40,42]$

$$
\bar{\Psi}=\left(\hat{\gamma}^{0} \Psi\right)^{+}=(\bullet)^{+} \hat{\gamma}^{0} \Psi,
$$

where $(\bullet)^{+}$means that the symbol $\bullet$ must be replaced by the element that the Hermitian conjugation operator acts on.

Formulas (2) for gamma operators were obtained by specifying the conjugation of a spinor by Formula (11). However, the most general form of the generalized Dirac conjugation, which ensures the Lorentz covariance of the transformation of conjugate spinors, is given by the formula

$$
\bar{\Psi}=(M \Psi)^{+},
$$

where $M$ is an operator that is constructed of gamma operators [42]. Moreover, for a space with signature $(p, q)$

$$
\begin{aligned}
& M=\eta_{+}+\eta_{-}, \\
& \eta_{+}=M_{i_{1} \ldots i_{p}} \hat{\gamma}_{+}^{i_{1}} \ldots \hat{\gamma}_{+}^{i_{p}}, \\
& \eta_{-}=M_{i_{1} \ldots i_{q}} \hat{\gamma}_{-}^{i_{1}} \ldots \hat{\gamma}_{-}^{i_{q}},
\end{aligned}
$$

where there is no summation over indices, $M_{i_{1} \ldots i_{p}+}$ and $M_{i_{1} \ldots i_{p}-}$ are numerical coefficients, $\hat{\gamma}_{+}^{i_{1}} \ldots \hat{\gamma}_{+}^{i_{p}}$ is the product of gamma operators with positive signature, and $\hat{\gamma}_{-}^{i_{1}} \ldots \hat{\gamma}_{-}^{i_{q}}$ is the product of gamma operators with negative signature [42].

It was proven in the theory of Krein spaces $[46,47]$ that the condition $M=\eta_{+}$must be satisfied for a four-dimensional spacetime with signature $(1,-1,-1,-1)$ for an invariant form $f$ of the spinor space, which provides the convolution of the spinors $\Psi_{1}$ and $\Psi_{2}$

$$
f=\left(M \Psi_{1}, \Psi_{2}\right) .
$$

Therefore, we refine the result of [42] and obtain $M=\hat{\gamma}^{0}$.

Infinitesimal transformations of basis spinors and their conjugate spinors, preserving relations (7) and (8) of the CAR-algebra, in the case of a four-dimensional spacetime, lead to the transformations of the field operators $\Psi$

$$
\Psi \prime=\left(1+i \hat{\gamma}^{a} d \omega_{a}+\frac{1}{4} \hat{\gamma}^{a b} d \omega_{a b}\right) \Psi,
$$


where $a, b=0,1,2,3,4,6,7$, transformation parameters $d \omega_{a}$ and $d \omega_{a b}=-d \omega_{b a}$ are real, and $\hat{\gamma}^{a b}=\hat{\gamma}^{a} \hat{\gamma}^{b}=-\hat{\gamma}^{b} \hat{\gamma}^{a}=\left[\gamma^{a b}, \bullet\right], a \neq b$ [42]. In (15), in the case of conjugation (11), two additional gamma operators $\hat{\gamma}^{6}$ and $\hat{\gamma}^{7}$ with negative signature appear in comparison with the Dirac theory [42]

$$
\begin{aligned}
& \hat{\gamma}^{6}=i \int \mathrm{d}^{3} p\left[\frac{\partial}{\partial \theta^{1}(p)} \frac{\partial}{\partial \theta^{2}(p)}+\theta^{2}(p) \theta^{1}(p)-\frac{\partial}{\partial \theta^{3}(p)} \frac{\partial}{\partial \theta^{4}(p)}-\theta^{4}(p) \theta^{3}(p), \bullet\right], \\
& \hat{\gamma}^{7}=\int \mathrm{d}^{3} p\left[\frac{\partial}{\partial \theta^{1}(p)} \frac{\partial}{\partial \theta^{2}(p)}-\theta^{2}(p) \theta^{1}(p)+\frac{\partial}{\partial \theta^{3}(p)} \frac{\partial}{\partial \theta^{4}(p)}-\theta^{4}(p) \theta^{3}(p), \bullet\right] .
\end{aligned}
$$

Operators $\hat{\gamma}^{a}, a=0,1,2,3,6,7$ form a basis in the space of Clifford vectors of the algebra, which we call the small Clifford algebra [40].

Operators $\hat{\gamma}^{5}$ and $\hat{\gamma}^{4}=i \hat{\gamma}^{5}$ are expressed via the product of other gamma operators in accordance with Formula (17)

$$
\hat{\gamma}^{0} \hat{\gamma}^{1} \hat{\gamma}^{2} \hat{\gamma}^{3} \hat{\gamma}^{6} \hat{\gamma}^{7}=i \hat{\gamma}^{4}=-\hat{\gamma}^{5}
$$

Lorentz boost operator

$$
\Lambda=\exp \left(\hat{\gamma}^{0 k} \varphi_{k}\right)=\exp \left(\hat{\gamma}^{0} \hat{\gamma} \varphi\right), k=1,2,3,
$$

changes the 4 -vector $\hat{\gamma}^{0} m$ to the 4 -vector

$$
\hat{\gamma}^{\mu} p_{\mu}=\hat{\gamma}^{0} p_{0}+\hat{\gamma} p=\exp \left(\hat{\gamma}^{0} \hat{\gamma} \varphi\right) \hat{\gamma}^{0} \exp \left(-\hat{\gamma}^{0} \hat{\gamma} \varphi\right) m=\exp \left(2 \hat{\gamma}^{0} \hat{\gamma} \varphi\right) \hat{\gamma}^{0} m,
$$

which corresponds to the spatial momentum $p$. In (19), $\varphi$ is the Lorentz boost angle, and $\hat{\gamma}$ is the spatial direction vector to which the vector $\hat{\gamma}^{0}$ is rotated.

Lorentz boost $\exp \left(\hat{\gamma}^{0 k} \varphi_{k}\right)$ changes the operators of annihilation $b_{\alpha}(0)=\frac{\partial}{\partial \theta^{\alpha}(0)}$ and creation $\bar{b}_{\alpha}(0)=\theta^{\alpha}(0)$ of a spinor with the momentum 0 to the operators of annihilation $b_{\alpha}(p)$ and creation $\bar{b}_{\alpha}(p)$ of a spinor with the momentum $p$ [41].

$$
\begin{aligned}
& b_{\alpha}(p)=\left.\exp \left(\hat{\gamma}^{0 k} \varphi_{k}\right) \frac{\partial}{\partial \theta^{\alpha}(0)}\right|_{0 \rightarrow p}=\left(\cosh \varphi(p)+\hat{\gamma}^{0} \hat{\gamma} \sinh \varphi(p)\right) \frac{\partial}{\partial \theta^{\alpha}(p)} \\
& \bar{b}_{\alpha}(p)=\left.\exp \left(\hat{\gamma}^{0 k} \varphi_{k}\right) \theta^{\alpha}(0)\right|_{0 \rightarrow p}=\left(\cosh \varphi(p)+\hat{\gamma}^{0} \hat{\gamma} \sinh \varphi(p)\right) \theta^{\alpha}(p) .
\end{aligned}
$$

Hereinafter, we consider active Lorentz transformations. Note that with passive Lorentz transformations, only the frame of reference changes. Therefore, in the new frame of reference, the components of the vectors and spinors change, but these quantities themselves remain unchanged. However, with active Lorentz transformations, the direction of the vectors changes, and the spinors and tensors change accordingly. In this case, we can assume that the frame of reference rotates together with vectors, spinors, and tensors. In the rotated frame of reference, the components of vectors, spinors and tensors have the same values as in the original one. However, in the original frame of reference, the components of vectors, spinors, and tensors change in the same way as in the case of passive Lorentz transformations. The momentum values in (22) are indicated in the initial frame of reference. Therefore, for all the quantities depending on momentum, the value 0 corresponding to the rest frame must be replaced by $p$ after rotation.

The creation $\bar{b}_{1}(p), \bar{b}_{2}(p)$ and the annihilation $b_{1}(p), b_{2}(p)$ operators of spinors and the creation $\bar{b}_{3}(p), \bar{b}_{4}(p)$ and annihilation $b_{3}(p), b_{4}(p)$ operators of antispinors are constructed of the generators of the large Clifford algebra using the Lorentz transformations in the small Clifford algebra. They also satisfy the canonical anticommutation relations

$$
\begin{aligned}
& \left\{\bar{b}_{l}(p), b_{k}\left(p^{\prime}\right)\right\}=\delta_{k}^{l} \delta\left(p-p^{\prime}\right), \\
& \left\{b_{k}(p), b_{l}\left(p^{\prime}\right)\right\}=\left\{\bar{b}_{k}(p), \bar{b}_{l}\left(p^{\prime}\right)\right\}=0 .
\end{aligned}
$$

The Lorentz covariance of the theory is directly related to the fulfillment of these relations. It should be noted that, in quantum mechanics, the creation and annihilation operators are usually considered as Hermitian conjugated. However, in the algebra of 
second quantization, Hermitian conjugation is not covariant with respect to the Lorentz transformations. In the theory we are considering, the bilinear form $\bar{\Psi}_{1} \Psi_{2}$ of the Dirac theory of spinors corresponds to the bilinear form $\left\{\bar{\Psi}_{1}, \Psi_{2}\right\}$ [42]. It gives the Lorentz scalar. Therefore, in it, only the anticommutator of the spinor and the Dirac conjugated spinor, which gives the Lorentz scalar, and not the anticommutator of the spinor and the Hermitian conjugated spinor, can be Lorentz-covariant.

Operator

$$
\hat{Q}=i \hat{\gamma}^{6} \hat{\gamma}^{7}
$$

is a generator of rotations in the plane of the axes $\hat{\gamma}^{6}, \hat{\gamma}^{7}$. Wherein

$$
\begin{aligned}
& \hat{Q} \Psi=\Psi, \\
& \hat{Q} \bar{\Psi}=-\bar{\Psi} .
\end{aligned}
$$

Operator $\hat{Q}$ displaces the average electric charge of the spinor multiplet from the zero level and, therefore, is the spinor hypercharge operator. In the considered case of four independent Grassmann variables, it is the operator of the electric charge $[43,44]$.

Transformations (15) preserve the CAR-algebra relations. In this case, rotations in the planes specified by the vectors $\hat{\gamma}^{\mu}, \mu=0,1,2,3$ and $\hat{\gamma}^{6}, \hat{\gamma}^{7}, \hat{\gamma}^{4}$ are prohibited, since otherwise, spacetime would have dimensions greater than four, and we are considering the case of a four-dimensional space. Therefore, in the expansion (15), the terms corresponding to such rotations must be equal to zero. Rotations in planes specified by the vectors $\hat{\gamma}^{6}, \hat{\gamma}^{7}$ and $\hat{\gamma}^{4}$ generate internal degrees of freedom of spinors $[43,44]$.

\section{Spinor Vacuum as an Alternative to the Dirac Sea}

The action of the annihilation operator $b_{1}(p)$ on the vacuum state vector $\Psi_{\mathrm{V}}$ should give zero. The only nonzero element in the algebra, multiplying by which $b_{1}(p)$ gives zero, is $b_{1}(p) \Psi_{\mathrm{V} 1}$, where $\Psi_{\mathrm{V} 1}$ is arbitrary factor. It means that $\Psi_{\mathrm{V}}=b_{1}(p) \Psi_{\mathrm{V} 1}$. In the theory of algebraic spinors, the spaces of spinors arise when the elements of the algebra are multiplied by the Hermitian idempotent. Therefore, it is necessary to construct $\Psi_{\mathrm{V}}$ in the form of a Hermitian idempotent. According to formulas (21), this is possible only if $\Psi_{\mathrm{V}}=b_{1}(p) \bar{b}_{1}(p) \Psi_{\mathrm{V} 2}$, where $\Psi_{\mathrm{V} 2}$ is a factor commuting with $b_{1}(p) \bar{b}_{1}(p)$. Similarly, we obtain

$$
\begin{aligned}
& \Psi_{\mathrm{V}}(p)=b_{1}(p) \bar{b}_{1}(p) b_{2}(p) \bar{b}_{2}(p) b_{3}(p) \bar{b}_{3}(p) b_{4}(p) \bar{b}_{4}(p), \\
& \Psi_{\mathrm{V}}=\Psi_{\mathrm{V}}(p) \Psi_{\mathrm{V} 3},
\end{aligned}
$$

where $\Psi_{\mathrm{V} 3}$ is some factor.

We considered the annihilation operators $b_{i}(p)$ for an arbitrary momentum $p$. Therefore, $\Psi_{\mathrm{V}}$ must contain factors $\Psi_{\mathrm{V}}(p)$ of all momentum values. For a continuous momentum spectrum, this is difficult. Therefore, we discretizede the momentum space in accordance with $[40,41]$ and considerd a quasi-continuous momentum spectrum. We divided the momentum space into a grid of cells of physically infinitesimal volumes $\Delta^{3} p_{i}$, and assigned a discrete momentum value to each cell. Basis spinors are generators of the CAR algebra generated by the discretization of the anticommutation relations (7), (8) and (21). In this case, Dirac delta function $\delta\left(p-p^{\prime}\right)$ must be replaced by a discrete approximation, and canonical anticommutation relations of the annihilation and creation operators become

$$
\begin{gathered}
\left\{b_{k}\left(p_{i}\right), \bar{b}_{l}\left(p_{j}\right)\right\}=\delta_{k}^{l} \frac{1}{\Delta^{3} p_{i}} \delta_{j}^{i} \\
\left\{b_{k}\left(p_{i}\right), b_{l}\left(p_{j}\right)\right\}=\left\{\bar{b}_{k}\left(p_{i}\right), \bar{b}_{l}\left(p_{j}\right)\right\}=0 .
\end{gathered}
$$

Further, throughout the formulas, to increase the readability of the formulas, continuous values are used. However, they denote discrete quasi-continuous values. 
The vacuum state vector [40]

$$
\Psi_{\mathrm{V}}=\prod_{i} \Psi_{\mathrm{V}}\left(p_{i}\right)
$$

is given via the factors $\Psi_{\mathrm{V}}\left(p_{i}\right)$ local in the momentum space

$$
\Psi_{\mathrm{V}}\left(p_{i}\right)=\left(\Delta^{3} p_{i}\right)^{4} b_{1}\left(p_{i}\right) \bar{b}_{1}\left(p_{i}\right) b_{2}\left(p_{i}\right) \bar{b}_{2}\left(p_{i}\right) b_{3}\left(p_{i}\right) \bar{b}_{3}\left(p_{i}\right) b_{4}\left(p_{i}\right) \bar{b}_{4}\left(p_{i}\right) .
$$

It is important that $b_{l}\left(p_{i}\right)$ and $\bar{b}_{l}\left(p_{i}\right)$ do not commute and do not anticommute. Therefore, the order of the factors $b_{l}\left(p_{i}\right)$ and $\bar{b}_{l}\left(p_{i}\right)$ in (28) cannot be changed. Factors $b_{<k>}\left(p_{i}\right) \bar{b}_{<k>}\left(p_{i}\right)$ commute with $b_{l}\left(p_{i}\right)$ and $\bar{b}_{l}\left(p_{i}\right)$ for all $k \neq l$ (hereinafter there is no summation over the indices in angle brackets). Therefore, taking into account (25)-(26), we obtain

$$
\begin{gathered}
b_{1}\left(p_{i}\right) \Psi_{\mathrm{V}}=b_{1}\left(p_{i}\right) \Psi_{\mathrm{V}}\left(p_{i}\right) \ldots=0, \\
\Psi_{1}\left(p_{i}\right)=\bar{b}_{1}\left(p_{i}\right) \Psi_{\mathrm{V}}=\bar{b}_{1}\left(p_{i}\right) \Psi_{\mathrm{V}}\left(p_{i}\right) \ldots=\left(\Delta^{3} p_{i}\right)^{4} \bar{b}_{1}\left(p_{i}\right) b_{1}\left(p_{i}\right) \bar{b}_{1}\left(p_{i}\right) b_{2}\left(p_{i}\right) \ldots \\
=\left(\Delta^{3} p_{i}\right)^{4}\left(\delta_{1}^{1} \frac{1}{\Delta^{3} p_{i}} \delta_{i}^{i}-b_{1}\left(p_{i}\right) \bar{b}_{1}\left(p_{i}\right)\right) \bar{b}_{1}\left(p_{i}\right) b_{2}\left(p_{i}\right) \ldots=\left(\Delta^{3} p_{i}\right)^{3} \bar{b}_{1}\left(p_{i}\right) b_{2}\left(p_{i}\right) \ldots \neq 0 . \\
\Delta^{3} p_{i} b_{1}\left(p_{i}\right) \Psi_{1}\left(p_{i}\right)=\Delta^{3} p_{i}\left(\delta_{1}^{1} \frac{1}{\Delta^{3} p_{i}} \delta_{i}^{i}-\bar{b}_{1}\left(p_{i}\right) b_{1}\left(p_{i}\right)\right) \Psi_{\mathrm{V}}=\Psi_{\mathrm{V}} .
\end{gathered}
$$

The annihilation operator $b_{1}\left(p_{i}\right)$ nullifies the vacuum, and the creation operator $\bar{b}_{1}\left(p_{i}\right)$ creates a one-particle state with momentum $p_{i}$ when it acts on the vacuum. Similar formulas are valid for operators $b_{k}\left(p_{i}\right)$ and $\bar{b}_{k}\left(p_{i}\right)$ with all possible values of $k$ and $p_{i}$.

For $p=0$ the expression of a factor $\Psi_{\mathrm{V}}\left(p_{i}\right)$ is

$$
\Psi_{\mathrm{V}}(0)=\left(\left.\Delta^{3} p\right|_{p=0}\right)^{4} \frac{\partial}{\partial \theta^{1}(0)} \theta^{1}(0) \frac{\partial}{\partial \theta^{2}(0)} \theta^{2}(0) \frac{\partial}{\partial \theta^{3}(0)} \theta^{3}(0) \frac{\partial}{\partial \theta^{4}(0)} \theta^{4}(0) .
$$

$\Psi_{\mathrm{V}}\left(p_{i}\right)$ we obtain from $\Psi_{\mathrm{V}}(0)$ by the Lorentz boost (18) of each of the factors in (32). It follows from (25) and (20) that

$$
\begin{aligned}
& \left(b_{<l>}\left(p_{i}\right) \bar{b}_{<l>}\left(p_{i}\right)\right)^{+} \\
& =\left(\hat{\gamma}^{0} \exp \left(\hat{\gamma}^{0 k} \varphi_{k}\right) \frac{\partial}{\partial \theta^{<l>}(0)}\right)\left(\exp \left(\hat{\gamma}^{0 j} \varphi_{j}\right) \frac{\partial}{\partial \theta_{<l>}(0)}\right)^{+} \\
& =\left(\exp \left(\hat{\gamma}^{-0 k} \varphi_{k}\right) \hat{\gamma}^{0} \frac{\partial}{\partial \theta^{<l>}(0)}\right)\left(\exp \left(-\hat{\gamma}^{0 j} \varphi_{j}\right) \theta^{<l>}(0)\right) \\
& =\left.\left(\exp \left(\hat{\gamma}^{-0 k} \varphi_{k}\right) \frac{\partial}{\partial \theta^{<l>}(0)}\right)\left(\exp \left(-\hat{\gamma}^{0 j} \varphi_{j}\right) \theta^{<l>}(0)\right)\right|_{p=0 \rightarrow p=-p_{i}} \\
& =b_{<l>}\left(-p_{i}\right) \bar{b}_{<l>}\left(-p_{i}\right) .
\end{aligned}
$$

There are both factors $b_{<l>}\left(p_{i}\right) \bar{b}_{<l>}\left(p_{i}\right)$ and factors $b_{<l>}\left(-p_{i}\right) \bar{b}_{<l>}\left(-p_{i}\right)$ in $\Psi_{\mathrm{V}}$. By virtue of (25) and (26), they commute with all other factors. Therefore,

$$
\Psi_{\mathrm{V}}^{+}=\Psi_{\mathrm{V}}
$$

Also, taking into account (25) and (26), it is easy to prove that

$$
\left(\Psi_{\mathrm{V}}\right)^{2}=\Psi_{\mathrm{V}}
$$

Thus, the vacuum operator $\Psi_{\mathrm{V}}$ is a Hermitian idempotent, and superalgebraic spinors, according to the theory of algebraic spinors, are algebraic spinors.

We have a countable infinite number of nonequivalent vacua. It suffices to change in the formula (27) in $\Psi_{\mathrm{V}}\left(p_{i}\right)$ the order of $b_{\alpha}\left(p_{i}\right)$ and $\bar{b}_{\alpha}\left(p_{i}\right)$ in an arbitrary number of factors $b_{\alpha}\left(p_{i}\right) \bar{b}_{\alpha}\left(p_{i}\right)$ to obtain a new vacuum. Such vacuum state vectors $\Psi_{\mathrm{V}}^{\prime}$ are algebraically completely equivalent if we consider the changed order $b_{\alpha}\left(p_{i}\right)$ as creation operators and the corresponding operators $\bar{b}_{\alpha}\left(p_{i}\right)$ as annihilation operators. In full accordance with [45], we have an infinite number of different vacuum state vectors, only for one of which the 
operator of the number of particles makes sense for a given set of creation and annihilation operators. However, specifying these vacuum state vectors in an explicit form allowed us to discover the meaningfulness of all of them if we did not fix in advance whether the generators of CAR algebras are creation operators or annihilation operators. Vacua differ only in the role of these generators.

The Hilbert space, scalar product, and generalized Dirac conjugation (11) define the Hermitian form and the indefinite inner product of spinors on the Hilbert space, corresponding to the theory of Clifford algebras and spinors [46-48]. Such spaces are called Krein spaces $[46,47]$.

Vacuum (27) is an algebraic realization of the concept of the Dirac Sea, free from the drawbacks of the idea of the presence of a "sea" of spinors with negative energy. The eigenvalues of the energy-momentum, spin operators, and electrical charge are equal to zero for vacuum (27). Therefore, there are no problems of infinite values of the Dirac Sea.

\section{R-Operators, Alternative Vacuum and Reversal Operator}

We consider active linear transformations of Clifford vectors

$$
V^{\prime}=A X A^{-1}
$$

in this case, the spinor transforms as

$$
\Psi^{\prime}=A \Psi
$$

The action of the operator (15) of infinitesimal transformations $1+d \hat{G}=1+[d G, \bullet]$ on the field operator $\Psi_{1} \Psi_{2} \ldots \Psi_{k}$ of the k-particle state is given by the formula

$$
\begin{aligned}
& (1+d \hat{G}) \Psi_{1} \Psi_{2} \ldots \Psi_{k}=1+\left[d G, \Psi_{1}\right] \Psi_{2} \ldots \Psi_{k}+\Psi_{1}\left[d G, \Psi_{2}\right] \ldots \Psi_{k}+\ldots \\
& =\left(e^{d \hat{G}} \Psi_{1}\right)\left(e^{d \hat{G}} \Psi_{2}\right) \ldots\left(e^{d \hat{G}} \Psi_{k}\right)
\end{aligned}
$$

where the parentheses limit the scope of the operators $e^{d \hat{G}}$. It generalizes the result of [41] to spaces of arbitrary even dimension.

Integration of (38) gives the result of the action of $e^{\hat{G}}$ on the $\Psi_{1} \Psi_{2} \ldots \Psi_{k}$

$$
e^{\hat{G}} \Psi_{1} \Psi_{2} \ldots \Psi_{k}=\left(e^{\hat{G}} \Psi_{1}\right)\left(e^{\hat{G}} \Psi_{2}\right) \ldots\left(e^{\hat{G}} \Psi_{k}\right) .
$$

Operator $R_{\hat{G}}=e^{\hat{G}}$ simultaneously transforms all factors in (39). We will call such operators R-operators.

Consider the effect of the boost on the vacuum state vector (27). To do this, first consider action of the boost

$$
\Lambda_{1}=\exp \left(\hat{\gamma}^{0 j} \alpha_{j}\right)
$$

on the factor $b_{<l>}\left(p_{i}\right) \bar{b}_{<l>}\left(p_{i}\right)$ in (28). Taking into account (20), we obtain

$$
b_{<l>}\left(p_{i}\right) \bar{b}_{<l>}\left(p_{i}\right)=\left(\exp \left(\hat{\gamma}^{0 k} \varphi_{k}\right) \frac{\partial}{\partial \theta^{<l>}(0)}\right)\left(\exp \left(\hat{\gamma}^{0 m} \varphi_{m}\right) \theta^{<l>}(0)\right) .
$$

The Lorentz transformation is an R-operator. According to (39), operator $\Lambda_{1}$ acts on each spinor factor in $b_{<l>}\left(p_{i}\right) \bar{b}_{<l>}\left(p_{i}\right)$. From (39)-(41) it follows

$$
\begin{aligned}
& \Lambda_{1} b_{<l>}\left(p_{i}\right) \bar{b}_{<l>}\left(p_{i}\right)=\left(\Lambda_{1} b_{<l>}\left(p_{i}\right)\right)\left(\Lambda_{1} \bar{b}_{<l>}\left(p_{i}\right)\right) \\
& =\left(\exp \left(\hat{\gamma}^{0 j} \alpha_{j}\right) \exp \left(\hat{\gamma}^{0 k} \varphi_{k}\right) \frac{\partial}{\partial \theta^{<l>}(0)}\right)\left(\exp \left(\hat{\gamma}^{0 j} \alpha_{j}\right) \exp \left(\hat{\gamma}^{0 m} \varphi_{m}\right) \theta^{<l>}(0)\right) \\
& =\left(\exp \left(\hat{\gamma}^{0 k} \lambda_{k}\right) \frac{\partial}{\partial \theta^{<l>}(0)}\right)\left(\exp \left(\hat{\gamma}^{0 m} \lambda_{m}\right) \theta^{<l>}(0)\right)=b_{<l>}\left(p_{s}\right) \bar{b}_{<l>}\left(p_{s}\right)
\end{aligned}
$$

where $\lambda_{j}$ are the real parameters (angles) of the resulting boost, and $p_{s}$ is the spatial momentum corresponding to this boost. Thus, the Lorentz transformation transforms the factor (28), corresponding to the momentum $p_{i}$, into the factor corresponding to the 
momentum $p_{s}$. In (27), this factor is replaced by a factor that previously corresponded to a different momentum, and this applies to all factors in (27). Factors corresponding to different momentums commute. Therefore, their product does not change as a result of the Lorentz transformation, and vacuum (27) does not change.

Consider unitary infinitesimal operator

$$
e^{d \hat{G}}=1+\hat{\gamma}^{05} d \omega
$$

where $d \omega$ is an infinitesimal real parameter. With respect to the action of $e^{d \hat{G}}$, spinor $\Psi$ and conjugated spinor $\bar{\Psi}$ transform as

$$
\begin{aligned}
& \Psi^{\prime}=\left(1+\hat{\gamma}^{05} d \omega\right) \Psi, \\
& \overline{\Psi^{\prime}}=\left(1-\hat{\gamma}^{05} d \omega\right) \bar{\Psi}
\end{aligned}
$$

The results of integration of transformations (44) for the final angle $\varphi=\frac{\pi}{2}$ are

$$
\begin{aligned}
& \Psi^{\prime}=\hat{\gamma}^{05} \Psi=R_{\hat{\gamma}^{05}} \Psi, \\
& \overline{\Psi^{\prime}}=-\hat{\gamma}^{05} \bar{\Psi}=R_{-\hat{\gamma}^{05}} \bar{\Psi} .
\end{aligned}
$$

We obtain from (45)

$$
\begin{aligned}
& \hat{\gamma}^{0 \prime}=R_{\hat{\gamma}^{05}} \hat{\gamma}^{0}\left(R_{\hat{\gamma}^{05}}\right)^{-1}=R_{-\hat{\gamma}^{05}} \hat{\gamma}^{0}\left(R_{-\hat{\gamma}^{05}}\right)^{-1}=-\hat{\gamma}^{0}, \\
& \hat{\gamma}^{5 \prime}=R_{\hat{\gamma}^{05}} \hat{\gamma}^{5}\left(R_{\hat{\gamma}^{05}}\right)^{-1}=R_{-\hat{\gamma}^{05}} \hat{\gamma}^{5}\left(R_{-\hat{\gamma}^{05}}\right)^{-1}=-\hat{\gamma}^{5} \text {, } \\
& \hat{\gamma}^{a \prime}=R_{\hat{\gamma}^{05}} \hat{\gamma}^{a}\left(R_{\hat{\gamma}^{05}}\right)^{-1}=R_{-\hat{\gamma}^{05}} \hat{\gamma}^{a}\left(R_{-\hat{\gamma}^{05}}\right)^{-1}=\hat{\gamma}^{a}, a=1,2,3,6,7 \text {. }
\end{aligned}
$$

Thus, operator $R_{\text {Racah }}=\hat{\gamma}^{05}$ for single-particle states and

$$
R_{\text {Racah }}=R_{\hat{\gamma}^{05}}
$$

in the common case is a time reflection operator.

We will call this operator Racah time reflection operator. Time reflection operator $\gamma_{D}^{0} \gamma_{D}^{5}$ was proposed by G. Racah in 1937 [49].

Let us consider the action of the operator $R_{ \pm \hat{\gamma}^{05}}$ on the vacuum state vector (32).

$$
\begin{aligned}
& R_{ \pm \hat{\gamma}^{05}} \Psi_{\mathrm{V}}(0)=\Psi_{\mathrm{altV}}(0) \\
& \Psi_{\mathrm{altV}}(0)=\left(\left.\Delta^{3} p\right|_{p=0}\right)^{4} \theta^{1}(0) \frac{\partial}{\partial \theta^{1}(0)} \theta^{2}(0) \frac{\partial}{\partial \theta^{2}(0)} \theta^{3}(0) \frac{\partial}{\partial \theta^{3}(0)} \theta^{4}(0) \frac{\partial}{\partial \theta^{4}(0)}
\end{aligned}
$$

Similarly,

$$
\begin{aligned}
& R_{ \pm \hat{\gamma}^{05}} \Psi_{\mathrm{V}}\left(p_{i}\right)=\Psi_{\mathrm{altV}}\left(p_{i}\right) \\
& R_{ \pm \hat{\gamma}^{05}} \Psi_{\mathrm{V}}=\Psi_{\mathrm{altV}} \\
& \Psi_{\mathrm{altV}}=\prod_{i} \Psi_{\mathrm{altV}}\left(p_{i}\right) .
\end{aligned}
$$

where $\Psi_{\mathrm{altV}}$ is an alternative vacuum. In $\Psi_{\mathrm{altV}}$ the order of factors (creation and annihilation operators) in each $\Psi_{\mathrm{V}}\left(p_{i}\right)$ is reversed

$$
\Psi_{\mathrm{altV}}\left(p_{i}\right)=\left(\Delta^{3} p_{i}\right)^{4} \bar{b}_{1}\left(p_{i}\right) b_{1}\left(p_{i}\right) \bar{b}_{2}\left(p_{i}\right) b_{2}\left(p_{i}\right) \bar{b}_{3}\left(p_{i}\right) b_{3}\left(p_{i}\right) \bar{b}_{4}\left(p_{i}\right) b_{4}\left(p_{i}\right) .
$$

This vacuum is invariant with respect to the Lorentz transformations and can be regarded as physical. However, in accordance with (50) and (51), in the Universe with vacuum (50), the role of creation and annihilation operators changes: operators $\bar{b}_{k}\left(p_{i}\right)$ are not creation operators, as for the Universe with vacuum (27), but annihilation operators. Additionally, operators $b_{k}\left(p_{i}\right)$ are not the annihilation operators, but the creation operators.

Thus, operator $R_{ \pm \hat{\gamma}^{05}}$ leads to the replacement of vacuum by an alternative one, which is not equivalent to the vacuum $\Psi_{\mathrm{V}}$. 
Consider the action of $R_{\hat{\gamma}^{a}}, a=1,2,3,4,6,7$ on $\Psi_{\mathrm{V}}$. Let us take the action of $R_{\hat{\gamma}^{7}}$ first as an example. Since $\hat{\gamma}^{7}$ commutes with the Lorentz transformation operator (40), acting on the factors $b_{<l>}\left(p_{i}\right) \bar{b}_{<l>}\left(p_{i}\right)$ included in $\Psi_{\mathrm{V}}\left(p_{i}\right)$ in (28), we obtain

$$
\begin{aligned}
& R_{\hat{\gamma}^{7}}\left(b_{1}\left(p_{i}\right) \bar{b}_{1}\left(p_{i}\right)\right)=\left(\hat{\gamma}^{7} b_{1}\left(p_{i}\right)\right)\left(\hat{\gamma}^{7} \bar{b}_{1}\left(p_{i}\right)\right) \\
& =\left(\hat{\gamma}^{7} \exp \left(\hat{\gamma}^{0 k} \varphi_{k}\right) \frac{\partial}{\partial \theta^{1}(0)}\right)\left(\hat{\gamma}^{7} \exp \left(\hat{\gamma}^{0 j} \varphi_{j}\right) \theta^{1}(0)\right) \\
& =\left(\exp \left(\hat{\gamma}^{0 k} \varphi_{k}\right) \hat{\gamma}^{7} \frac{\partial}{\partial \theta^{1}(0)}\right)\left(\exp \left(\hat{\gamma}^{0 j} \varphi_{j}\right) \hat{\gamma}^{7} \theta^{1}(0)\right) \\
& =\left(-\exp \left(\hat{\gamma}^{0 k} \varphi_{k}\right) \theta^{2}(0)\right)\left(-\exp \left(\hat{\gamma}^{0 j} \varphi_{j}\right) \frac{\partial}{\partial \theta^{2}(0)}\right) \\
& =\bar{b}_{2}\left(p_{i}\right) b_{2}\left(p_{i}\right) .
\end{aligned}
$$

Similarly,

$$
\begin{aligned}
& R_{\hat{\gamma}^{7}}\left(b_{2}\left(p_{i}\right) \bar{b}_{2}\left(p_{i}\right)\right)=\bar{b}_{1}\left(p_{i}\right) b_{1}\left(p_{i}\right), \\
& R_{\hat{\gamma}^{7}}\left(b_{3}\left(p_{i}\right) \bar{b}_{3}\left(p_{i}\right)\right)=\bar{b}_{4}\left(p_{i}\right) b_{4}\left(p_{i}\right), \\
& R_{\hat{\gamma}^{7}}\left(b_{4}\left(p_{i}\right) \bar{b}_{4}\left(p_{i}\right)\right)=\bar{b}_{3}\left(p_{i}\right) b_{3}\left(p_{i}\right) .
\end{aligned}
$$

Thus,

$$
\begin{aligned}
& R_{\hat{\gamma}^{7}} \Psi_{\mathrm{V}}\left(p_{i}\right)=\Psi_{\mathrm{altV}}\left(p_{i}\right), \\
& R_{\hat{\gamma}^{7}} \Psi_{\mathrm{V}}=\Psi_{\text {altV }} .
\end{aligned}
$$

We obtain the results for $R_{\hat{\gamma}^{6}}$ and $R_{i \hat{\gamma}^{5}}$ in exactly the same way.

There is some difference from the previous cases for operators $R_{\hat{\gamma}^{k}}, k=1,2,3$, since they do not commute with the Lorentz transformation.

$$
\begin{aligned}
& R_{\hat{\gamma}^{1}}\left(b_{1}\left(p_{i}\right) \bar{b}_{1}\left(p_{i}\right)\right)=\left(\hat{\gamma}^{1} b_{1}\left(p_{i}\right)\right)\left(\hat{\gamma}^{1} \bar{b}_{1}\left(p_{i}\right)\right) \\
& =\left(\exp \left(-\hat{\gamma}^{01} \varphi_{1}+\hat{\gamma}^{02} \varphi_{2}+\hat{\gamma}^{03} \varphi_{3}\right) \hat{\gamma}^{1} \frac{\partial}{\partial \theta^{1}(0)}\right) \\
& \left(\exp \left(-\hat{\gamma}^{01} \varphi_{1}+\hat{\gamma}^{02} \varphi_{2}+\hat{\gamma}^{03} \varphi_{3}\right) \hat{\gamma}^{1} \theta^{1}(0)\right) \\
& =\left(-\exp \left(\hat{\gamma}^{0 j} \varphi_{j}{ }^{\prime}\right) \theta^{4}(0)\right)\left(-\exp \left(\hat{\gamma}^{0 l} \varphi_{l}{ }^{\prime}\right) \frac{\partial}{\partial \theta^{4}(0)}\right) \\
& =\bar{b}_{4}\left(p_{i}{ }^{\prime}\right) b_{4}\left(p_{i}{ }^{\prime}\right),
\end{aligned}
$$

where $\varphi_{j}{ }^{\prime}$ and $p_{i}{ }^{\prime}$ correspond to the momentum for which the axis $\hat{\gamma}^{1}$ component has changed its sign. We obtain similar formulas for all factors $b_{<k>}\left(p_{i}\right) \bar{b}_{<k>}\left(p_{i}\right)$ in (28). Therefore,

$$
R_{\hat{\gamma}^{1}} \Psi_{\mathrm{V}}\left(p_{i}\right)=\Psi_{\mathrm{altV}}\left(p_{i}^{\prime}\right) .
$$

That is, operator $R_{\hat{\gamma}^{7}}$ simply swaps the commuting factors in (50). Therefore,

$$
R_{\hat{\gamma}^{1}} \Psi_{\mathrm{V}}=\Psi_{\mathrm{altV}} .
$$

The proof is completely similar for $R_{\hat{\gamma}^{2}}$ and $R_{\hat{\gamma}^{3}}$. Thus, operators $R_{\hat{\gamma}^{1}}, R_{\hat{\gamma}^{2}}, R_{\hat{\gamma}^{3}}, R_{\hat{\gamma}^{6}}$, $R_{\hat{\gamma}^{7}}, R_{i \hat{\gamma}^{5}}$ transform the vacuum $\Psi_{V}$ into $\Psi_{\text {altV }}$. In the same way, it is proven that they transform $\Psi_{\text {altV }}$ into $\Psi_{V}$.

Recall that all operators must preserve CAR. Therefore, only operators $R_{i \hat{\gamma}^{0}}$ and $R_{i \hat{\gamma}^{5}}$ make sense, not $R_{\hat{\gamma}^{0}}$ and $R_{\hat{\gamma}^{5}}$. Additionally, since $i \hat{\gamma}^{0} i \hat{\gamma}^{5}=-\hat{\gamma}^{0} \hat{\gamma}^{5}=-\hat{\gamma}^{05}$, operator $R_{\hat{\gamma}^{0}}$ makes sense.

It is easy to check that $\Psi_{\text {altV }}$ is invariant with respect to the Lorentz transformations, Hermitian conjugation, spatial reflection, operator $R_{i \hat{\gamma}}{ }^{0}$ action, and other transformations that leave the vacuum $\Psi_{V}$ invariant.

Consider the transposition operator $(\bullet)^{T}$. It changes the order of all factors $\frac{\partial}{\partial \theta^{a}(p)}$, $\theta^{b}\left(p^{\prime}\right)$ and converts $\frac{\partial}{\partial \theta^{a}(p)}$ to $\theta^{a}(p)$ and $\theta^{b}\left(p^{\prime}\right)$ to $\frac{\partial}{\partial \theta^{b}\left(p^{\prime}\right)}$. It is easy to prove $[40,41]$ that

$$
\begin{aligned}
& (\bullet)^{T} \hat{\gamma}^{a} \Psi=-\hat{\gamma}^{a} \Psi^{T}, a=0,2,6,5, \\
& (\bullet)^{T} \hat{\gamma}^{b} \Psi=\hat{\gamma}^{b} \Psi^{T}, b=1,3,7 .
\end{aligned}
$$


Hence,

$$
\begin{aligned}
& (\bullet)^{T} \hat{\gamma}^{a}\left((\bullet)^{T}\right)^{-1} \Psi^{T}=-\hat{\gamma}^{a} \Psi^{T}, a=0,2,6,5, \\
& (\bullet)^{T} \hat{\gamma}^{b}\left((\bullet)^{T}\right)^{-1} \Psi^{T}=\hat{\gamma}^{b} \Psi^{T}, b=1,3,7 .
\end{aligned}
$$

Equation (59) mean that the transposition operator $(\bullet)^{T}$ reflects axes $\hat{\gamma}^{0}, \hat{\gamma}^{2}$, and $\hat{\gamma}^{6}$, as well as

, and does not change other basis vectors of the Clifford algebra. By multiplying the CAR-preserving operator $(\bullet)^{T}$ by the CAR-preserving reflection operator $\hat{\gamma}^{26}$, we obtain the CAR-preserving vacuum-preserving time reflection operator

$$
R_{\text {Schwinger }}=-(\bullet)^{T} \hat{\gamma}^{26}=-\hat{\gamma}^{26}(\bullet)^{T} \text {. }
$$

Formula (60) is valid only for the single-particle case. If there is a product of singleparticle operators, it must be replaced by

$$
R_{\text {Schwinger }}=R_{-\hat{\gamma}^{26}}(\bullet)^{T}=(\bullet)^{T} R_{-\hat{\gamma}^{26}} \text {. }
$$

As we will show below, operator $R_{\text {Schwinger }}$ is directly related to the unitary time inversion operator proposed by Schwinger [15].

When acting on single-particle field operators, action of the $R_{\text {Racah }}$ operator (48) coincides with the action of $R_{\text {Schwinger }}(61)$. They both are unitary and preserve CAR. However, operator $R_{\text {Racah }}$ changes the vacuum to an alternative one, but $R_{\text {Schwinger }}$ preserves usual vacuum state. Their difference is that, when acting on the product of field operators, $R_{\text {Racah }}$ does not change their order, but $R_{\text {Schwinger }}$ reverses the order of the factors. Therefore,

$$
R=R_{-1} R_{\text {Racah }} R_{\text {Schwinger }}=R_{\hat{\gamma}^{05}} R_{\hat{\gamma}^{26}}(\bullet)^{T}
$$

is reversal operator of the spinor field operators. Operator $R$ preserves CAR and does not change gamma operators or single-particle spinors. It commutes with all elements of the small Clifford algebra. However it reverses order of spinors in multi-particle field operators. In particular,

$$
\begin{aligned}
& R \Psi_{\mathrm{V}}=\Psi_{\mathrm{altV}} \\
& R \Psi_{\mathrm{altV}}=\Psi_{\mathrm{V}} .
\end{aligned}
$$

If there is any operator that transforms the vacuum into an alternative one, multiplying it by $R$ will give an operator that coincides with it when acting on the field operator of the single-particle state of the spinor, but preserves the vacuum.

\section{Spatial Inversion $P$}

Consider the case when $d \hat{G}=i \hat{\gamma}^{0} d \omega_{0}$. In this case, operator $e^{\hat{G}}$ is equal to

$$
e^{i \hat{\gamma}^{0} \omega_{0}}=\cos \omega_{0}+i \hat{\gamma}^{0} \sin \omega_{0}
$$

where $\omega_{0}$ is a real constant. Let us choose $\omega_{0}=\frac{\pi}{2}$. Then, we obtain operator $R_{i \hat{\gamma}^{0}}$, which acts on the single-particle state in the same way as the commutator $i \hat{\gamma}^{0}=\left[i \gamma^{0}, \bullet\right]$

$$
R_{i \hat{\gamma}^{0}} \Psi=i\left[\gamma^{0}, \Psi\right]
$$

Therefore, for single-particle states, $R_{i \hat{\gamma}^{0}}$ and $i \hat{\gamma}^{0}$ cannot be distinguished. However, when acting on the product of the field operators, $R_{i \hat{\gamma}^{0}}$ acts on each of the factors:

$$
R_{i \hat{\gamma}^{0}} \Psi_{1} \Psi_{2} \ldots \Psi_{k}=i\left[\gamma^{0}, \Psi_{1}\right] i\left[\gamma^{0}, \Psi_{2}\right] \ldots i\left[\gamma^{0}, \Psi_{k}\right]=\left(R_{i \hat{\gamma}^{0}} \Psi_{1}\right)\left(R_{i \hat{\gamma}^{0}} \Psi_{2}\right) \ldots\left(R_{i \hat{\gamma}^{0}} \Psi_{k}\right)
$$


Operator $R_{i \hat{\gamma}^{0}}$ is unitary. It transforms Clifford vectors as

$$
\begin{aligned}
& \hat{\gamma}^{0 \prime}=\hat{\gamma}^{0} \\
& \hat{\gamma}^{k \prime}=-\hat{\gamma}^{k}, k=1,2,3,6,7,4,
\end{aligned}
$$

where, here and below, we mark the transformed values with dashes.

Thus, operator $R_{i \hat{\gamma}^{0}}$ reflects all the basis vectors except $\hat{\gamma}^{0}$, including $\hat{\gamma}^{6}$ and $\hat{\gamma}^{7}$. Similarly, the reflection operator of spatial Clifford vectors $R_{i \hat{\gamma}^{0}} R_{\hat{\gamma}^{67}}=R_{\hat{\gamma}^{0} \hat{Q}}$ reflects only spatial basis vectors $\hat{\gamma}^{1}, \hat{\gamma}^{2}, \hat{\gamma}^{3}$ and, of course, also reflects pseudovector $\hat{\gamma}^{4}$. Operators $R_{i \hat{\gamma}^{0}}$ and $R_{\hat{\gamma}^{0} \hat{Q}}$ are similar but not equivalent. They are uniquely determined up to the sign. Factors other than \pm 1 are forbidden by CAR algebra relations.

We introduce symmetry operator $P$ of the spatial inversion in complete analogy with the scheme described by Bjorken and Drell [6], taking into account that the Dirac equation in superalgebraic form has the form [50]

$$
\hat{\gamma}^{\mu}\left(i \partial_{\mu}-q A_{\mu}\right) \Psi(t, x)=m \Psi(t, x)
$$

where $t=x^{0}$ is the time, $A_{\mu}$ is the vector potential, $q= \pm g$ is the value of the spinor charge, and $g$ is the interaction constant.

This equation can also be written as

$$
\hat{\gamma}^{\mu}\left(i \partial_{\mu}-g \hat{Q} A_{\mu}\right) \Psi(t, x)=m \Psi(t, x)
$$

Eigenvalues of operator $\hat{Q}$ are +1 for the spinor and -1 for the antispinor. Both (68) and (69) have the same solutions.

Hereinafter, in accordance with the established tradition, we call the discrete symmetry operators $P$ and $T$ the inversions, not the reflections. They are more complex than the reflection operators.

With respect to the action of operator $R_{\hat{\gamma}^{0} \hat{Q}^{\prime}}$ Equation (69) becomes

$$
\left(\hat{\gamma}^{0}\left(i \partial_{0}-g \hat{Q} A_{0}\right)-\hat{\gamma}^{k}\left(i \partial_{k}-g \hat{Q} A_{k}\right)\right) \Psi^{\prime}(t, x)=m \Psi^{\prime}(t, x) .
$$

In order for Equation (70) to take the form (68), it is necessary to carry out transformation $R_{-x^{k}}$ that changes the sign of the spatial coordinates $x^{k}$, their derivatives and the sign of the spatial part of the vector potential

, and does not change the zeros components of the quantities:

$$
\begin{aligned}
& x^{0 \prime}=R_{-x} x^{0}=x^{0}, x^{k \prime}=R_{-x} x^{k}=-x^{k}, \\
& \partial_{0}{ }^{\prime}=R_{-x} \partial_{0}=\partial_{0}, \partial_{k}{ }^{\prime}=R_{-x} \partial_{k}=-\partial_{k \prime} \\
& A_{0}^{\prime}=R_{-x} A_{0}=A_{0}, A_{k}^{\prime}=R_{-x} A_{k}=-A_{k} .
\end{aligned}
$$

Then the symmetry operators

$$
\begin{gathered}
P_{1}=R_{-x^{k}} R_{i \hat{\gamma}^{0}} \\
P=R_{-x^{k}} R_{i \hat{\gamma}^{0}} R_{\hat{\gamma}^{67}}=R_{-x^{k}} R_{\hat{\gamma}^{0} \hat{Q}}
\end{gathered}
$$

transform Equation (71) into the required form (74)

$$
\hat{\gamma}^{\mu}\left(i \partial_{\mu}{ }^{\prime}-g \hat{Q} A_{\mu}{ }^{\prime}\right) \Psi\left(t^{\prime}, x^{\prime}\right)=m \Psi\left(t^{\prime}, x^{\prime}\right) .
$$

Operator $R_{-x^{k}}$ changes the sign of the spatial part not only for coordinates $x^{k} \rightarrow-x^{k}$ and vector potential $A_{k} \rightarrow-A_{k}$ but in general for all vector quantities.

The same result can be obtained in a more convenient way, in fact, proposed by Lüders [4]. This is done in the following way. The Dirac equation (69) can be rewritten as

$$
i \partial_{0} \Psi(t, x)=\hat{H} \Psi(t, x)
$$




$$
\hat{H}=\hat{\gamma}^{0} m+g \hat{Q} A_{0}-\hat{\gamma}^{0} \hat{\gamma}^{k}\left(i \partial_{k}-g \hat{Q} A_{k}\right),
$$

where $\hat{H}$ is the Hamiltonian.

Any symmetry operator must commute with the Hamiltonian. Operator $R_{\hat{\gamma}^{0}} \hat{0}$ commutes with the first two terms in Hamiltonian (76), but anticommutes with the third term. For commutativity with it, the operator needs to add factor $R_{-x^{k}}$ that changes the sign of $\partial_{k}$ and $A_{k}$.

Already, Pauli noted that the transformation of the spinor components causes a change in the components of vectors and other tensor quantities [8]. This fact is easy to understand by considering the so-called bilinear covariants (covariant bilinear forms) $[6,11,14]$. In accordance with [50], traditional Dirac theory expressions for bilinear forms should be replaced by anticommutators that are similar to them in the superalgebraic representation of spinors (that is, in the algebra of second quantization).

The action of operator $R_{\hat{\gamma}^{0} \hat{Q}}$ leads to transformations of the spinors

$$
\begin{aligned}
& \Psi^{\prime}=\hat{\gamma}^{0} \hat{Q} \Psi=\hat{\gamma}^{0} \Psi, \\
& \overline{\Psi^{\prime}}=\left(\hat{\gamma}^{0} \hat{\gamma}^{0} \hat{Q} \Psi\right)^{+}=\Psi^{+},
\end{aligned}
$$

which, in turn, lead to the following rules for the transformations of scalars, 4-vectors, and tensors

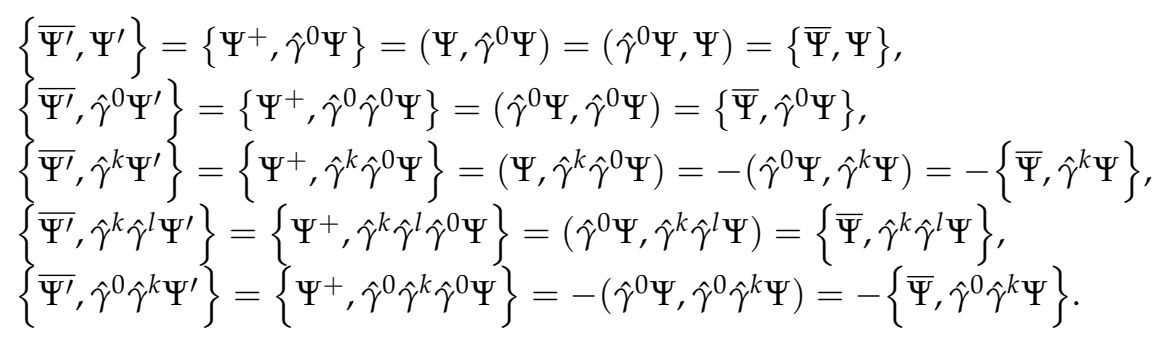

Consequently, in the case of spatial reflection, the scalar components, the spatial tensor components, and the zeros components of the vectors do not change. Additionally, the spatial components of the vectors and the mixed (with index $0 \mathrm{k}$ ) components of the second-order tensors change sign. Since transformation (77) does not affect charges, it refers to the components of all quantities of the corresponding form, both to vector quantities (coordinates $x^{\mu}$, vector potential $A^{\mu}$ of the electric field) and to mixed tensor, to which the momentum $P^{\mu}$ belongs (it is a component of the energy-momentum tensor $T^{0 \mu}$ ).

Thus, we obtain the usual formulas $[6,14]$ for the transformation of components of tensors of different ranks with respect to spatial inversion. We started our consideration with active transformations of Clifford vectors; however, the symmetry requirements led to the fact that, simultaneously with the basis Clifford vectors, it is necessary to change the components of the vectors. In this case, an arbitrary vector $\hat{\gamma}^{\mu} V_{\mu}$ is converted into the vector $\hat{\gamma}^{\mu \prime} V_{\mu}{ }^{\prime}=\hat{\gamma}^{\mu} V_{\mu}$. Therefore, the spatial inversion $P$ is reduced to changing the coordinate system. That is, it is an improper Lorentz transformation and is a passive transformation, such as the physically observed proper Lorentz transformations associated with the motion of the reference frame.

Consider the action of the spatial reflection $P$ on the vacuum. From (73) and (27)-(28), it follows

$$
P \Psi_{V}=R_{-x^{k}} R_{\hat{\gamma}^{0} \hat{Q}} \prod_{i} \Psi_{\mathrm{V}}\left(p_{i}\right)=\prod_{i} R_{\hat{\gamma}^{0} \hat{Q}} \Psi_{\mathrm{V}}\left(p_{i}\right) .
$$


Consider action of $R_{\hat{\gamma}^{0} \hat{Q}}$ on factors $b_{<l>}\left(p_{i}\right) \bar{b}_{<l>}\left(p_{i}\right)$ in $\Psi_{\mathrm{V}}\left(p_{i}\right)$. From (25)-(28), we obtain that

$$
\begin{aligned}
& R_{\hat{\gamma}^{0} \hat{Q}}\left(b_{<l>}\left(p_{i}\right) \bar{b}_{<l>}\left(p_{i}\right)\right)=\left(\hat{\gamma}^{0} \hat{Q} b_{<l>}\left(p_{i}\right)\right)\left(\hat{\gamma}^{0} \hat{Q} \bar{b}_{<l>}\left(p_{i}\right)\right) \\
& =-\left(\hat{\gamma}^{0} b_{<l>}\left(p_{i}\right)\right)\left(\hat{\gamma}^{0} \bar{b}_{<l>}\left(p_{i}\right)\right) \\
& =-\left(\hat{\gamma}^{0} \exp \left(\hat{\gamma}^{0} \varphi_{k}\right) \frac{\partial}{\partial \theta^{<l>}(0)}\right)\left(\hat{\gamma}^{0}\left(\hat{\gamma}^{0} \exp \left(\hat{\gamma}^{0 j} \varphi_{j}\right) \frac{\partial}{\partial \theta^{<l>}(0)}\right)^{+}\right) \\
& =\left(\exp \left(-\hat{\gamma}^{0 k} \varphi_{k}\right) \frac{\partial}{\partial \theta<l>(0)}\right)\left(\left(\hat{\gamma}^{0} \exp \left(-\hat{\gamma}^{0 j} \varphi_{j}\right) \frac{\partial}{\partial \theta^{<l>}(0)}\right)^{+}\right) \\
& =b_{<l>}\left(-p_{i}\right) \bar{b}_{<l>}\left(-p_{i}\right) .
\end{aligned}
$$

Therefore,

$$
R_{\hat{\gamma}^{0} \hat{Q}} \Psi_{\mathrm{V}}\left(p_{i}\right)=\Psi_{\mathrm{V}}\left(-p_{i}\right) .
$$

Since (27) contains both factors $\Psi_{\mathrm{V}}\left(p_{i}\right)$ and $\Psi_{\mathrm{V}}\left(-p_{i}\right)$, they change places with respect to the action of the operator $P$. However, all factors $\Psi_{\mathrm{V}}\left(p_{i}\right)$ for different $p_{i}$ commute since they include an even number of spinor factors. Consequently,

$$
P \Psi_{V}=\Psi_{V}
$$

That is, with respect to the action of the operator of spatial inversion $P$, the vacuum remains invariant.

\section{Time Inversion $T$}

Operator $T$ of the time inversion is antiunitary $[5,6,12,14,17]$. The derivation of the formula for it is carried out in exactly the same way as for $P$ (73).

Since $T$ is antiunitary, it must include the factor $(\bullet)^{*}$. Therefore, as a result of the action of operator $T$ on both sides of (75), we obtain

$$
-i\left(T \partial_{0}\right) T \Psi(t, x)=T \hat{H} \Psi(t, x) .
$$

The parentheses, as before, limit the scope of the operator $T$. In this case,

$$
\left(T \partial_{0}\right)=T \partial_{0} T^{-1}
$$

It follows from the time inversion requirement

$$
t^{\prime}=x^{\prime 0}=\left(T x^{0}\right)=-x^{0} .
$$

that operator $T$ must have factor $R_{-x^{0}}$ that changes the sign of component $t=x^{0}$ corresponding to the time. In this case, the relation

$$
\partial^{\prime}{ }_{0}=\left(T \partial_{0}\right)=-\partial_{0}
$$

should be fulfilled. Therefore,

$$
i \partial_{0}^{\prime} T \Psi(t, x)=T \hat{H} \Psi(t, x) .
$$

It follows from (87) that, for the equation of motion of the spinor (75) to be invariant with respect to this transformation, operator $T$ must commute with the Hamiltonian (76). Therefore, for a nonzero mass $m$, operator $T$ must commute with $\hat{\gamma}^{0}$. Since the basis space vectors $\hat{\gamma}^{1}, \hat{\gamma}^{2}, \hat{\gamma}^{3}$ must have the same commutation properties with $T$, they can be contained in $T$ only as $\hat{\gamma}^{2}(\bullet)^{*}$ or $\hat{\gamma}^{1} \hat{\gamma}^{3}(\bullet)^{*}$. Both of these variants preserve CAR. In addition to these factors, $T$ may contain CAR-preserving factors $i \hat{\gamma}^{0}$ and $i \hat{\gamma}^{5}$. We do not yet consider operators $\hat{\gamma}^{6}$ and $\hat{\gamma}^{7}$. Time inversion should lead to a change in the direction of the spin to the opposite. Since complex conjugation in the chosen representation of the gamma operators does not change the spin of the basis spinors (they are real), the spin 
projection operators $s^{k l}=\frac{i}{4}\left(\hat{\gamma}^{k} \hat{\gamma}^{l}-\hat{\gamma}^{l} \hat{\gamma}^{k}\right) ; k, l=1,2,3$ must change their sign. Operators $i \hat{\gamma}^{0}$ and $i \hat{\gamma}^{5}$ both commute with $s^{k l}$ and cannot influence the sign change. Operators $\hat{\gamma}^{2}(\bullet)^{*}$ and $\hat{\gamma}^{1} \hat{\gamma}^{3}(\bullet)^{*}$ anticommute with $s^{k l}$ and can be part of operator T. But factors $i \hat{\gamma}^{0}$ and $\hat{\gamma}^{2}(\bullet)^{*}$ change the chirality so they can only be together in the form $R_{-x^{0}} R_{i \hat{\gamma}^{0} \hat{\gamma}^{2}}(\bullet)^{*}$. Therefore, only three variants of operator $T$ are possible: $R_{-x^{0}} R_{\hat{\gamma}^{1} \hat{\gamma}^{3}}(\bullet)^{*}, R_{-x^{0}} R_{i \hat{\gamma}^{0} \hat{\gamma}^{2} i \hat{\gamma}^{5}}(\bullet)^{*}$, and $R_{-x^{0}} R_{i \hat{\gamma}^{0} \hat{\gamma}^{2}}(\bullet)^{*}$. However, the first and the second variants are equivalent to the symmetry operator $R_{i \hat{Q}}$, and we may not consider the second variant. Therefore, only variants $R_{-x^{0}} R_{\hat{\gamma}^{1} \hat{\gamma}^{3}}(\bullet)^{*}$ and $R_{-x^{0}} R_{i \hat{\gamma} \hat{\gamma}^{2}}(\bullet)^{*}$ remain. However, operator $R_{-x^{0}} R_{i \hat{\gamma}^{0} \hat{\gamma}^{2}}(\bullet)^{*}$ anticommutes with $\hat{\gamma}^{0}$ and, therefore, does not fit.

With respect to the action of operator $R_{-x^{0}} R_{\hat{\gamma}^{1} \hat{\gamma}^{3}}(\bullet)^{*}$, Equation (68) becomes

$$
\hat{\gamma}^{\mu}\left(i \partial_{\mu}{ }^{\prime}-\hat{Q} A_{\mu}{ }^{\prime}\right) \Psi^{\prime}(-t, x)=m \Psi^{\prime}(-t, x),
$$

where

$$
\begin{aligned}
& x^{0 \prime}=-x^{0}, x^{k \prime}=x^{k}, \\
& \partial_{0}{ }^{\prime}=-\partial_{0}, \partial_{k}{ }^{\prime}=\partial_{k \prime} \\
& A_{0}{ }^{\prime}==A_{0}, A_{k}{ }^{\prime}=-A_{k} .
\end{aligned}
$$

The formulas for $x^{\prime}{ }_{\mu}$ and $A^{\prime}{ }_{\mu}$ are derived from the requirement that operator $T$ commutes with the Hamiltonian. Thus, the symmetry operator

$$
T_{1}=R_{-x^{0}} R_{\hat{\gamma}^{1} \hat{\gamma}^{3}}(\bullet)^{*}
$$

transforms Equation (68) into the required form (91)

$$
\hat{\gamma}^{\mu}\left(i \partial_{\mu}{ }^{\prime}-\hat{Q} A_{\mu}{ }^{\prime}\right) \Psi\left(t^{\prime}, x^{\prime}\right)=m \Psi\left(t^{\prime}, x^{\prime}\right) .
$$

The result (90) in the single-particle form corresponds to [5,6,14,17].

However, operator $T$ must "rewind the film". That is, it changes the direction of motion and spin of the spinor without changing the chirality, but it should not change the spinor to the conjugated one or the spinor annihilation operator to the antispinor creation operator. Therefore, for example, $T$ should replace the basis spinor $\frac{\partial}{\partial \theta^{1}(p)}$ by $\frac{\partial}{\partial \theta^{2}(-p)}$. Operator $T_{1}$ in (90) provides this. It follows from the properties of gamma operators (2), (16) and the requirement to preserve CAR that, while preserving vacuum, formula (90) is unambiguous up to the sign in front of $\hat{\gamma}^{1} \hat{\gamma}^{3}$. However, when "rewinding the film", the annihilation operator must become the creation one, and vice versa. Therefore, $T_{1}$ is not suitable as a time inversion operator, and for this operator, it is necessary to multiply it by the reversal operator (62). As the result, we obtain

$$
T=R T_{1}=R R_{-x^{0}} R_{\hat{\gamma}^{1} \hat{\gamma}^{3}}(\bullet)^{*}=R_{-x^{0}} R_{\hat{\gamma}^{7}}(\bullet)^{+} .
$$

Consider the result of the action of operator $T$ on the vacuum. Taking into account (34), we obtain

$$
T \Psi_{V}=R_{-x^{0}} R_{\hat{\gamma}^{7}}(\bullet)^{+} \Psi_{V}=R_{\hat{\gamma}^{7}} \Psi_{V}^{+}=R_{\hat{\gamma}^{7}} \Psi_{V}
$$

It follows from (93) and (54) that

$$
T \Psi_{V}=\Psi_{\mathrm{altV}}
$$

Thus, operator $T$ changes the vacuum to an alternative one and cannot be the operator of the exact symmetry of the system and the operator of the symmetry of the vacuum.

Operators $R_{\text {Racah }}(48)$ and $R_{\text {Schwinger }}(61)$ are unitary. They commute with $i \partial_{0}$ and, for a nonzero mass $m$, anticommute with the first term in Hamiltonian (76). Therefore, they cannot preserve the invariance of the equation of motion (75) and cannot be the symmetry operators. 
Let us set operators

$$
\begin{aligned}
& T_{\text {Racah }}=R_{-x^{0}} R_{\text {Racah }}=R_{-x^{0}} R_{\hat{\gamma}^{05}}, \\
& T_{\text {Schwinger }}=R_{-x^{0}} R_{\text {Schwinger }}=R_{-x^{0}} R_{\hat{\gamma}^{26}}(\bullet)^{T} .
\end{aligned}
$$

They commute with Hamiltonian (76) under the conditions

$$
\begin{aligned}
& x^{0 \prime}=-x^{0}, x^{k \prime}=x^{k} \\
& \partial_{0}{ }^{\prime}=-\partial_{0}, \partial_{k}{ }^{\prime}=\partial_{k} \\
& A_{0}{ }^{\prime}=-A_{0}, A_{k}{ }^{\prime}=A_{k} .
\end{aligned}
$$

Hence, these are unitary time inversion operators. Consider how the operator $T_{\text {Racah }}$ transforms scalars

$$
\left\{\overline{\Psi^{\prime}}, \Psi^{\prime}\right\}=\left(\hat{\gamma}^{0} \hat{\gamma}^{0} \hat{\gamma}^{5} \Psi, \hat{\gamma}^{0} \hat{\gamma}^{5} \Psi\right)=\left(\hat{\gamma}^{0} \hat{\gamma}^{5} \Psi, \hat{\gamma}^{5} \Psi\right)=\left(\hat{\gamma}^{5} \hat{\gamma}^{0} \hat{\gamma}^{5} \Psi, \Psi\right)=-\{\bar{\Psi}, \Psi\} .
$$

Thus, $T_{\text {Racah }}$ does not preserve 4 -scalar values. In particular, it violates relations (21) of the CAR algebra of creation and annihilation operators. After all, the form (14), where $M=\eta_{+}=\hat{\gamma}^{0}$, can be invariant only for the Lorentz transformations of the fourdimensional spacetime. However, operator $T_{\text {Racah }}$ corresponds to rotation in the fivedimensional spacetime with the signature $(1,1,-1,-1,-1)$. It should retain the form (14) invariant in the case $M=\eta_{+}=\hat{\gamma}^{0} \hat{\gamma}^{5}$.

For operator $T_{\text {Schwinger }}$ we obtain exactly the same result since, for single-particle states, it coincides with $T_{\text {Racah }}$ and differs in the order of the factors for multi-particle states.

\section{Charge Conjugation $C$}

$C P T$ operator must be antiunitary $[5,20,22]$. Operator $P$ is unitary; $T$ is antiunitary. Therefore, charge conjugation operator $C$ must be unitary. Using similar reasoning as for $P$ and $T$, we obtain that preserving vacuum $\Psi_{V}$ charge conjugation operator is

$$
C_{1}=R_{-q} R_{i \hat{\gamma}^{56}}
$$

There must be antiparticles in the Universe that can be obtained from particles using $C P T$ transformation. In this case, the vacuum after the action of the CPT operator should be $\Psi_{V}$ and not $\Psi_{\text {altV }}$. If we replace operator $P$ by operator $R P$, Berestetskii theorem [11] is violated, which states that the internal spatial parity of a system of a fermion and an antifermion is -1 . For example,

$$
\begin{gathered}
P \frac{\partial}{\partial \theta^{1}(0)} \frac{\partial}{\partial \theta^{3}(0)}=\left(\hat{\gamma}^{0} \hat{Q} \frac{\partial}{\partial \theta^{1}(0)}\right)\left(\hat{\gamma}^{0} \hat{Q} \frac{\partial}{\partial \theta^{3}(0)}\right)=-\frac{\partial}{\partial \theta^{1}(0)} \frac{\partial}{\partial \theta^{3}(0)}, \\
R P \frac{\partial}{\partial \theta^{1}(0)} \frac{\partial}{\partial \theta^{3}(0)}=-\frac{\partial}{\partial \theta^{3}(0)} \frac{\partial}{\partial \theta^{1}(0)}=\frac{\partial}{\partial \theta^{1}(0)} \frac{\partial}{\partial \theta^{3}(0)} .
\end{gathered}
$$

Thus, in the case of operator $P$, the result corresponds to the Berestetskii theorem, but in the case of $R P$, we obtain the contradiction.

Therefore, it is the charge conjugation operator $C$, and not the spatial reflection operator $P$, that should replace the vacuum with an alternative one. Hence,

$$
C=R C_{1}=R R_{-q} R_{i \hat{\gamma}^{56}}=R_{-q} R_{-i \hat{\gamma}^{02}}(\bullet)^{T} .
$$

Consider the action of operator $C$ on basis spinors $b_{\alpha}(p) e^{-i p_{\mu} x^{\mu}}$ and $\bar{b}_{\tau}(p) e^{i p_{\mu} x^{\mu}}$,obtained from spinors $b_{\alpha}(0) e^{-\left.i m x^{0}\right|_{p=0}}$ and $\bar{b}_{\tau}(0) e^{\left.i m x^{0}\right|_{p=0}}$ using boost $e^{\hat{\gamma}^{0} \hat{\gamma} \varphi}$. Since $\hat{\gamma}^{02}(\bullet)^{T}$ commutes with $e^{\hat{\gamma}^{0} \hat{\gamma} \varphi}$, we obtain 


$$
\begin{aligned}
& C b_{1}(p) e^{-i p_{\mu} x^{\mu}}=-i \hat{\gamma}^{02}(\bullet)^{T} e^{\hat{\gamma}^{0} \hat{\gamma} \varphi} \frac{\partial}{\partial \theta^{1}(0)} e^{-i p_{\mu} x^{\mu}}=-e^{\hat{\gamma}} \hat{\gamma} \varphi i \hat{\gamma}^{02}(\bullet)^{T} \frac{\partial}{\partial \theta^{1}(0)} e^{-i p_{\mu} x^{\mu}}=b_{4}(p) e^{-i p_{\mu} x^{\mu}}, \\
& C b_{2}(p) e^{-i p_{\mu} x^{\mu}}=-i \hat{\gamma}^{02}(\bullet)^{T} e^{\hat{\gamma}^{0} \hat{\gamma} \varphi} \frac{\partial}{\partial \theta^{2}(0)} e^{-i p_{\mu} x^{\mu}}=-e^{\hat{\gamma}^{0}} \hat{\gamma} \varphi_{i} \hat{\gamma}^{02}(\bullet)^{T} \frac{\partial}{\partial \theta^{2}(0)} e^{-i p_{\mu} x^{\mu}}=-b_{3}(p) e^{-i p_{\mu} x^{\mu}} \text {, } \\
& C \bar{b}_{3}(p) e^{i p_{\mu} x^{\mu}}=-i \hat{\gamma}^{02}(\bullet)^{T} e^{\hat{\gamma}^{0} \hat{\gamma} \varphi} \theta^{3}(0) e^{i p_{\mu} x^{\mu}}=-e^{\hat{\gamma}^{0} \hat{\gamma} \varphi} i \hat{\gamma}^{02}(\bullet)^{T} \theta^{3}(0) e^{i p_{\mu} x^{\mu}}=\bar{b}_{2}(p) e^{i p_{\mu} x^{\mu}} \text {, } \\
& C \bar{b}_{4}(p) e^{i p_{\mu} x^{\mu}}=-i \hat{\gamma}^{02}(\bullet)^{T} e^{\hat{\gamma}^{0} \hat{\gamma} \varphi} \theta^{4}(p) e^{i p_{\mu} x^{\mu}}=-e^{\hat{\gamma}^{0} \hat{\gamma} \varphi} i \hat{\gamma}^{02}(\bullet)^{T} \theta^{4}(0) e^{i p_{\mu} x^{\mu}}=-\bar{b}_{1}(p) e^{i p_{\mu} x^{\mu}} .
\end{aligned}
$$

It can be seen from (102) that operator $C$ does not change the momentum and the spin of the field operators but changes basis spinors to basis antispinors. In this case, the positive-frequency terms remain positive-frequency, and the negative-frequency ones remain negative-frequency.

Consider how the operator of charge conjugation $[5,6,17]$

$$
C_{\text {holes }}=-R_{-q} i \hat{\gamma}^{2}(\bullet)^{*}
$$

acts in the theory of "holes". Since $i \hat{\gamma}^{2}(\bullet)^{*}$ commutes with $e^{\hat{\gamma} \hat{\gamma} \varphi}$, we obtain

$$
\begin{aligned}
& C_{\text {holes }} b_{1}(p) e^{-i p_{\mu} x^{\mu}}=-i \hat{\gamma}^{2}(\bullet)^{*} e^{\hat{\gamma} \hat{\gamma} \varphi} \frac{\partial}{\partial \theta^{1}(0)} e^{-i p_{\mu} x^{\mu}}=-e^{\hat{\gamma}^{0} \hat{\gamma} \varphi} i \hat{\gamma}^{2}(\bullet)^{*} \frac{\partial}{\partial \theta^{1}(0)} e^{-i p_{\mu} x^{\mu}}=-\bar{b}_{4}(p) e^{i p_{\mu} x^{\mu}} \text {, }
\end{aligned}
$$

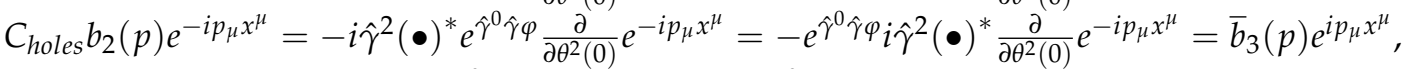

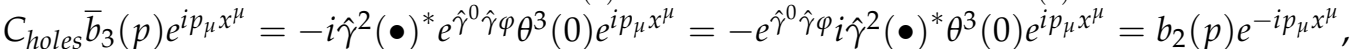

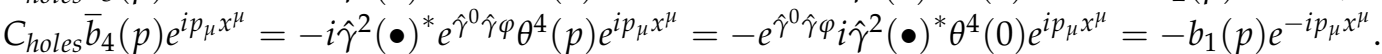

Note that operators $\bar{b}_{j}(p) e^{i p_{\mu} x^{\mu}}$ correspond to momentum $-p$, not $p$, since $i \partial_{k} \bar{b}_{j}(p) e^{i p_{\mu} x^{\mu}}=$ $-p_{k} \bar{b}_{j}(p) e^{i p_{\mu} x^{\mu}}$. As a result of the action of operator $C_{\text {holes }}$, the positive-frequency terms of the spinor field transform into negative-frequency terms of the same spinor but with the opposite momentum and spin. If we consider these terms as the "holes" in the Dirac Sea, they correspond to the positive-frequency terms of the antispinor. With such replacement, the results (104) fully agree with the results (102) obtained without using the concept of the Dirac Sea. In this case, operator $C_{\text {holes }}$ of charge conjugation in the theory of "holes", as already mentioned, is antiunitary. However, if we assume that the replacement of the "holes" in the Dirac Sea by antiparticles is operator $R_{\text {sea }}$, we obtain

$$
C=R_{\text {sea }} C_{\text {holes }}
$$

where $R_{\text {sea }}$ is the antiunitary operator, which changes the momentum and the projection of the spin of the spinor to the opposite ones and also changes the creation operator to the annihilation operator and the annihilation operator to the creation operator. Comparison of (105) to (102) and (104) implies that $R_{\text {sea }}$ is simply the generalized Dirac conjugation operator

$$
R_{\text {sea }}=(\bullet)^{+} \hat{\gamma}^{0},
$$

And, therefore,

$$
C=(\bullet)^{+} \hat{\gamma}^{0} C_{\text {holes }} .
$$

Thus, if the concept of charge conjugation in the theory of "holes" is formulated correctly, the operator of charge conjugation turns out to be unitary. That should be the case based on the antiunitary nature of the CPT transformation.

Let us now consider the action of operator of charge conjugation $C$ on the field operators of the spinor-antispinor system using the example of positronium at the ground state [11,12]. Orthopositronium has the spin $J=1$ and the charge parity of -1 . Parapositronium has the spin $J=0$ and the charge parity of +1 . If the projections of the spins $J_{3}$ and $J^{\prime}{ }_{3}$ are directed along the $\mathrm{z}$ axis, the wave functions $\psi_{\text {para }}\left(p, p^{\prime}, J_{3}, J^{\prime}{ }_{3}\right)$ and $\psi_{\text {orto }}\left(p, p^{\prime}, J_{3}, J^{\prime}{ }_{3}\right)$ satisfy the relations

$$
\begin{aligned}
& \psi_{\text {orto }}\left(p, p^{\prime}, J_{3}, J^{\prime}{ }_{3}\right)=\psi_{\text {orto }}\left(p^{\prime}, p, J_{3}, J^{\prime}{ }_{3}\right)=\psi_{\text {orto }}\left(p, p^{\prime}, J^{\prime}{ }_{3}, J_{3}\right) \\
& \psi_{\text {para }}\left(p, p^{\prime}, J_{3}, J^{\prime}{ }_{3}\right)=\psi_{\text {para }}\left(p^{\prime}, p, J_{3}, J^{\prime}{ }_{3}\right)=-\psi_{\text {para }}\left(p, p^{\prime}, J^{\prime}{ }_{3}, J_{3}\right) .
\end{aligned}
$$


For the orthopositronium with the spin projection +1 in the nonrelativistic case, where the negative-frequency components are negligible, we obtain

$$
\begin{aligned}
& \Psi_{\text {orto },+1}=\iint d^{3} p d^{3} p^{\prime} \psi_{\text {orto }}\left(p, p^{\prime}, \frac{1}{2}, \frac{1}{2}\right)\left(\frac{\partial}{\partial \theta^{1}(p)} \frac{\partial}{\partial \theta^{4}\left(p^{\prime}\right)}+\frac{\partial}{\partial \theta^{1}\left(p^{\prime}\right)} \frac{\partial}{\partial \theta^{4}(p)}\right) \\
& C \Psi_{\text {orto },+1}=\iint d^{3} p d^{3} p^{\prime} \psi_{\text {orto }}\left(p, p^{\prime}, \frac{1}{2}, \frac{1}{2}\right)\left(-\frac{\partial}{\partial \theta^{1}\left(p^{\prime}\right)} \frac{\partial}{\partial \theta^{4}(p)}-\frac{\partial}{\partial \theta^{1}(p)} \frac{\partial}{\partial \theta^{4}\left(p^{\prime}\right)}\right) \\
& =-\Psi_{\text {orto },+1} .
\end{aligned}
$$

For orthopositronium with a spin projection of -1 , everything turns out exactly the same way. For the orthopositronium with spin projection 0 , we obtain

$$
\begin{aligned}
& \Psi_{\text {orto }, 0}=\iint d^{3} p d^{3} p^{\prime} \psi_{\text {orto }}\left(p, p^{\prime}, \frac{1}{2},-\frac{1}{2}\right)\left(\frac{\partial}{\partial \theta^{1}(p)} \frac{\partial}{\partial \theta^{3}\left(p^{\prime}\right)}+\frac{\partial}{\partial \theta^{1}\left(p^{\prime}\right)} \frac{\partial}{\partial \theta^{3}(p)}\right. \\
& \left.+\frac{\partial}{\partial \theta^{4}(p)} \frac{\partial}{\partial \theta^{2}\left(p^{\prime}\right)}+\frac{\partial}{\partial \theta^{4}\left(p^{\prime}\right)} \frac{\partial}{\partial \theta^{2}(p)}\right) \\
& C \Psi_{\text {orto }, 0}=-\Psi_{\text {orto }, 0} .
\end{aligned}
$$

Similarly, for the parapositronium (spin 0 , projection of spin 0 ), we obtain

$$
\begin{aligned}
& \Psi_{\text {para }}=\iint d^{3} p d^{3} p^{\prime} \psi_{\text {para }}\left(p, p^{\prime}, \frac{1}{2},-\frac{1}{2}\right)\left(\frac{\partial}{\partial \theta^{1}(p)} \frac{\partial}{\partial \theta^{3}\left(p^{\prime}\right)}+\frac{\partial}{\partial \theta^{1}\left(p^{\prime}\right)} \frac{\partial}{\partial \theta^{3}(p)}\right. \\
& \left.-\frac{\partial}{\partial \theta^{4}(p)} \frac{\partial}{\partial \theta^{2}\left(p^{\prime}\right)}-\frac{\partial}{\partial \theta^{4}\left(p^{\prime}\right)} \frac{\partial}{\partial \theta^{2}(p)}\right) \\
& C \Psi_{\text {para }}=\Psi_{\text {para }} .
\end{aligned}
$$

Thus, for the orthopositronium, the charge parity is -1 , and for the parapositronium, it is +1 . This corresponds to the known facts $[11,12]$. If we use the operator $C_{1}$ instead of $C$, the sign is opposite to the required one.

\section{The CPT Operator}

Using Formulas (101), (73), and (92), we obtain expression for operator $\Theta=C P T$

$$
\begin{gathered}
\Theta=R_{-q} R_{-x^{\mu}} R_{\hat{\gamma}^{26}}(\bullet)^{*}=R_{-q} R_{-x^{\mu}} J_{+}, \\
J_{+}=R_{\hat{\gamma}^{26}}(\bullet)^{*},
\end{gathered}
$$

where $J_{+}$is the operator of the real structure ("charge conjugation") in the theory of Krein spaces $[46,47]$.

Note that Bizi, Brouder, and Besnard [46,47] understood charge conjugation as antiunitary CPT conjugation. However, at the same time, when considering the requirement of commutation of the operator of a real structure with the Dirac operator $D=i \gamma^{\mu} \partial_{\mu}$, they did not take into account that the CPT conjugation changes sign of the $\partial_{\mu}$ operator. Therefore, instead of the operator $J_{-}$, as indicated in $[46,47]$, it is necessary to use the operator $\Theta$. Moreover, in the operator $\Theta$ it is necessary to use the operator $J_{+}$, and not $J_{-}$, as an operator transforming the basis spinors. As shown by Pauli [8] and Jost [21], the CPT operator is the operator of the real structure of spinors.

The resulting operator $\Theta$ corresponds to vacuum $\Psi_{V}$ of our Universe and can be realized in it. The same applies to operators $P$ and $C T$.

Operator (112) $\Theta$ differs in form from its usual form in RQFT since it does not explicitly contain Hermitian conjugation. Let us change Formula (112) so that the Hermitian conjugation operator is explicitly present in it. It follows from (62) that

$$
R^{2}=1
$$

Therefore, formula (112) can be rewritten as

$$
\Theta=R R_{-q} R_{-x^{\mu}} R_{\hat{\gamma}^{05} \hat{\gamma}^{26}}(\bullet)^{T} R_{\hat{\gamma}^{26}}(\bullet)^{*}=R R_{-q} R_{-x^{\mu}} R_{-\hat{\gamma}^{5}}(\bullet)^{+} R_{\hat{\gamma}^{0}} .
$$

That is

$$
\Theta=R R_{-q} R_{-x^{\mu}} R_{-\hat{\gamma}^{5}} \overline{(\bullet)} .
$$


where $\overline{(\bullet)}=(\bullet)^{+} R_{\hat{\gamma}^{0}}$ is the generalized Dirac conjugation operator.

Formula (116) is unambiguous up to a phase factor \pm 1 .

\section{Discussion}

We have constructed a consistent algebraic theory of the second quantization of spinors based on the theory of superalgebraic spinors. In this theory, the main role is played by the CAR algebra of Grassmann densities and their derivatives.

Formulas (73), (92) and (103), (105)-(107) establish a connection between the formulas for the concept of "holes" in the Dirac Sea and the algebraic theory of spinors. The concept of the Dirac Sea made it possible to obtain formulas that correctly describe the properties of antiparticles and made a very large contribution to the development of the quantum field theory. However, the vacuum in it has not only an infinite charge and infinite negative mass but also infinite densities of these quantities. As a result of that, their fluctuations should lead to infinite forces acting on the spinors. There are no such problems in the theory of superalgebraic spinors. In this theory, the vacuum has zero eigenvalues of the operators of observable physical quantities. Charge conjugation in the theory of "holes" in the Dirac Sea contains the operation of transformation from a "hole" to an antiparticle. This operation must be matched with an antiunitary operator. Therefore, in the correct formulation, the antiunitary operator of charge conjugation in the theory of the Dirac Sea must be supplemented with an antiunitary factor $R_{\text {sea }}$, which is the operator of the generalized Dirac conjugation. The resulting operator (107) of the charge conjugation is unitary and coincides with the operator of charge conjugation of the theory of superalgebraic spinors.

The proposed approach made it possible to construct an explicit algebraic form (27) of the spinor vacuum $\Psi_{\mathrm{V}}$. This is one of an infinite number of possible vacua, which differ only in the role of the creation and annihilation operators for each of the possible values of the momentum. In all publications devoted to the $C, P$, and $T$ transformations of spinors, it was assumed that the vacuum is invariant under these transformations. We have proven that Lorentz transformations, as well as operators $P, C T$ and $C P T$, leave the vacuum $\Psi_{\mathrm{V}}$ invariant. However, operators $C$ and $T$ transform vacuum $\Psi_{V}$ into an alternative one $\Psi_{\text {alt }}$ and, therefore, cannot be realized in our Universe. Corresponding symmetries must be violated by processes associated with the difference in the action of operators $C$ and $C_{1}$, as well as $T$ and $T_{1}$, on states of physical systems. This difference, due to the properties of the reverse operator (62), does not manifest itself for single-particle states. Therefore, interaction with vacuum $\Psi_{V}$ is slightly different from interaction with alternative vacuum $\Psi_{\text {alt }}$. Consequently, the symmetry breaking should be small for the cases when the spinor can be regarded as an isolated particle.

The presence of vacua differing in the action of the creation and annihilation operators on them is similar to what happens in curved spacetime, where the vacuum is not invariant under the Lorentz transformations. In this case, each frame of reference corresponds to its own vacuum [51]. Moreover, in such frames of reference, each moment of time corresponds to its own vacuum [52]. However, in our case, we have only two spinor vacua, $\Psi_{V}$ and $\Psi_{\text {altv }}$. This is the case only for small Clifford algebra. In large Clifford algebra, there are operators that can transform different vacua into each other. Perhaps such transformations correspond to curved spacetime. This issue requires further research.

Funding: This research received no external funding.

Conflicts of Interest: The author declares no conflict of interest.

\section{References}

1. Kramers, H.A. Théorie générale de la rotation paramagnétique dans les cristaux. Proc. Kon. Neer. Akad. Wet. 1930, 33,959-972.

2. Wigner, E. Über die Operation der Zeitumkehr in der Quantenmechanik. In Nachrichten von der Gesellschaft der Wissenschaften zu Göttingen; Mathematisch-Physikalische Klasse; Weidmannsche Buchhandlung: Berlin, Germany, 1932; pp. 546-559.

3. Kramers, H.A. The use of charge-conjugated wave-functions in the hole-theory of the electron. Proc. Kon. Neer. Akad. Wet. 1937, 40, 814-823.

4. $\quad$ Lüders, G. Proof of the TCP theorem. Ann. Phys. 1957, 2, 1-15. [CrossRef] 
5. Grawert, G.; Lüders, G.; Rollnik, H. The TCP theorem and its applications. Fortschr. Phys. 1959, 7, 291-328. [CrossRef]

6. Bjorken, J.D.; Drell, S.D. Relativistic Quantum Mechanics; Mcgraw-Hill: New York, NY, USA, 1964.

7. Schwinger, J. The Theory of Quantized Fields. II. Phys. Rev. 1953, 91, 713-728. [CrossRef]

8. Pauli, W. Exclusion principle, Lorentz group and reflection of space-time and charge. In Niels Bohr and the Development of Physics: Essays Dedicated to Niels Bohr on the Occasion of His Seventieth Birthday; Pergamon Press: London, UK, $1955 ;$ pp. 30-51.

9. Bell, J.S. Time reversal in field theory. Proc. R. Soc. A 1955, 231, 479-495.

10. Bjorken, J.D.; Drell, S.D. Relativistic Quantum Fields; Mcgraw-Hill: New York, NY, USA, 1965.

11. Berestetskii, V.B.; Lifshitz, E.M.; Pitaevskii, L.P. Quantum Electrodynamics: Volume 4; Butterworth-Heinemann: Oxford, UK, 1982.

12. Weinberg, S. The Quantum Theory of Fields, Volume 1: Foundations; Cambridge University Press: Cambridge, UK, 1995. [CrossRef]

13. Matthews, P.T. The Relativistic Quantum Theory of Elementary Particle Interactions. Lectures Given by P. T. Matthews. Notes Compiled by F. Fujii, No. NYO-2097; Rochester University: Rochester, NY, USA, 1957.

14. Peskin, M.E.; Schroeder, D.V. An Introduction to Quantum Field Theory; Addison-Wesley Publishing Company: Boston, MA, USA, 1995. [CrossRef]

15. Schwinger, J. The Theory of Quantized Fields. I. Phys. Rev. 1951, 82, 914-927. [CrossRef]

16. Lüders, G. On the equivalence of invariance under time reversal and under particle-antiparticle conjugation for relativistic field theories. Dan. Mat. Fys. Medd. 1954, 28, 1-17.

17. Schwabl, F. Advanced Quantum Mechanics; Springer: Berlin/Heidelberg, Germany, 2008. [CrossRef]

18. Landau, L. On the conservation laws for weak interactions. Nucl. Phys. 1957, 3, 127-131. [CrossRef]

19. Epstein, H. CTP Invariance of the S-Matrix in a Theory of Local Observables. J. Math. Phys. 1967, 8, 750-767. [CrossRef]

20. Jost, R. Eine Bemerkung zum CTP-theorem. Helv. Phys. Acta 1957, 30, 409-416.

21. Jost, R. Das Pauli-prinzip und die Lorentz-gruppe. In Theoretical Physics in the Twentieth Century. A Memorial Volume to Wolfgang Pauli; Fierz, M., Weisskopf, V.F., Eds.; Interscience Publishers: Cambridge, UK; New York, NY, USA, 1960; pp. $107-136$.

22. Streater, R.F.; Wightman, A.S. PCT. Spin and Statistics, and All That; WA Benjamin: New York, NY, USA, 1964. [CrossRef]

23. Wess, J. The CPT-theorem and its significance for fundamental physics. Hyperfine Interact. 1989, 44, 3-8. [CrossRef]

24. Greenberg, O.W. Why is CPT Fundamental? Found. Phys. 2006, 36, 1535-1553. [CrossRef]

25. Christenson, J.H.; Cronin, J.W.; Fitch, V.L.; Turlay, R. Evidence for the $2 \pi$ Decay of the $K_{2}^{0}$ Meson. Phys. Rev. Lett. 1964, 13, 138-140. [CrossRef]

26. Bernabéu, J.; Segarra, A. Signatures of the genuine and matter-induced components of the CP violation asymmetry in neutrino oscillations. JHEP 2018, 2018, 63. [CrossRef]

27. Petcov, S.T.; Zhou, Y.-L. On neutrino mixing in matter and CP and T violation effects in neutrino oscillations. Phys. Lett. B 2018, 785, 95-104. [CrossRef]

28. Kostelecký, V.A.; Russell, N. Data tables for Lorentz and CPT violation. Rev. Mod. Phys. 2011, 83, 11-31. [CrossRef]

29. Karan, A. Dealing with $\mathrm{T}$ and $\mathrm{CPT}$ violations in mixing as well as direct and indirect $\mathrm{CP}$ violations for neutral mesons decaying to two vectors. Eur. Phys. J. C 2020, 80, 782. [CrossRef]

30. Lounesto, P. Clifford Algebras and Spinors; Cambridge University Press: Cambridge, UK, 2001. [CrossRef]

31. Lawson, H.; Michelsohn, M.-L. Spin Geometry; Princeton University Press: Princeton, NJ, USA, 1990. [CrossRef]

32. Borštnik, N.M. Spin connection as a superpartner of a vielbein. Phys. Lett. B 1992, 292, 25-29. [CrossRef]

33. Mankoš-Borštnik, N. Spinor and vector representations in four-dimensional Grassmann space. J. Math. Phys. 1993, 34, 3731-3745. [CrossRef]

34. Borštnik, N.M.; Nielsen, H.B.F. Understanding the second quantization of fermions in Clifford and in Grassmann space. New way of second quantization of fermions-Part I. Bled Workshops Phys. 2019, 20, 109-119.

35. Borštnik, N.M.; Nielsen, H.B.F. Understanding the second quantization of fermions in Clifford and in Grassmann space. New way of second quantization of fermions-Part II. Bled Workshops Phys. 2019, 20, 120-134.

36. Delanghe, R.; Sommen, F.; Souček, V. An explicit realization of spinor spaces and its application to Clifford analysis. Appl. Anal. 1992, 45, 95-115. [CrossRef]

37. Brackx, F.; De Schepper, H.; Souček, V. On the structure of complex Clifford algebra. Adv. Appl. Clifford Algebras 2011, 21, 477-492. [CrossRef]

38. Pavšic, M. A theory of quantized fields based on orthogonal and symplectic Clifford algebras. Adv. Appl. Clifford Algebras 2012, 22, 449-481. [CrossRef]

39. Monakhov, V.V. Construction of a fermionic vacuum and the fermionic operators of creation and annihilation in the theory of algebraic spinors. Phys. Part. Nucl. 2017, 48, 836-838. [CrossRef]

40. Monakhov, V. Vacuum and Spacetime Signature in the Theory of Superalgebraic Spinors. Universe 2019, 5, 162. [CrossRef]

41. Monakhov, V.V. Superalgebraic structure of Lorentz transformations. J. Phys. 2017, 1051, 012023. [CrossRef]

42. Monakhov, V.V. Generalization of Dirac Conjugation in the Superalgebraic Theory of Spinors. Theor. Math. Phys. 2019, 200, 1026-1042. [CrossRef]

43. Monakhov, V.V. Spacetime and inner space of spinors in the theory of superalgebraic spinors. J. Phys. 2020, 1557, 012031. [CrossRef]

44. Monakhov, V.V. Generation of Electroweak Interaction by Analogs of Dirac Gamma Matrices Constructed from Operators of the Creation and Annihilation of Spinors. Bull. Russ. Acad. Sci. Phys. 2020, 84, 1216-1220. [CrossRef] 
45. Gårding, L.; Wightman, A. Representations of the anticommutation relations. Proc. Nat. Acad. Sci. USA 1954, 40, 617-621. [CrossRef]

46. Bizi, N.; Brouder, C.; Besnard, F. Space and time dimensions of algebras with application to Lorentzian noncommutative geometry and quantum electrodynamics. J. Math. Phys. 2018, 59, 062303. [CrossRef]

47. Besnard, F.; Bizi, N. On the definition of spacetimes in noncommutative geometry: Part I. J. Geom. Phys. 2018, 123, 292-309. [CrossRef]

48. Robinson, P.L. Spinors and canonical hermitian forms. Glasg. Math. J. 1988, 30, 263-270. [CrossRef]

49. Racah, G. Sulla simmetria tra particelle e antiparticelle. Il Nuovo Cim. 1937, 14, 322-328. [CrossRef]

50. Monakhov, V.V. A Superalgebraic Form of the Dirac Equation. Bull. Russ. Acad. Sci. Phys. 2019, 83, 1173-1178. [CrossRef]

51. Birrell, N.D.; Davies, P.C.W. Quantum Fields in Curved Space; Cambridge University Press: Cambridge, UK, 1982. [CrossRef]

52. Grib, A.A.; Damaskinskiŭ, E.V.; Maksimov, V.M. The problem of symmetry breaking and invariance of the vacuum in quantum field theory. Sov. Phys. Uspekhi 1971, 13, 798-815. [CrossRef] 